\title{
Liquid-mesophase-solid transitions: Systematics of a density-wave theory
}

\author{
Mangal C. Mahato, M. Raj Lakshmi, Rahul Pandit, and H. R. Krishnamurthy \\ Department of Physics, Indian Institute of Science, Bangalore 560012, India
}

1987)

The density-wave theory of Ramakrishnan and Yussouff is used to study phase transitions between liquid, liquid-crystalline, and crystalline phases. The different phases considered are liquid, nematic, smectic, discotic, bcc plastic crystal, orientationally ordered bcc, and a new incommensurate bcc crystal with orientational order. The direct correlation function, required as an input for the theory, is expressed approximately in terms of five generalized Fourier coefficients. The theory is then used to obtain sections through the phase diagram in the five-dimensional space of these coefficients. Simple approximations for the direct correlation function of hard ellipsoids of revolution are used to compare these phase diagrams with those obtained from experiments and numerical simulations. Molecular-field theories of smectic and discotic ordering are reexamined, and, given the potentials they use, it is shown that an orientationally ordered bcc crystal has a lower free energy than either the smectic or the discotic phase. The conditions required to stabilize smectic and discotic phases are examined.

\section{INTRODUCTION}

There have been many theoretical studies of phase transitions from liquid to liquid-crystalline mesophases $^{l-3}$ and, over the past decade, of transitions from liquid to solid phases (crystals in most cases) using the density-functional approach. ${ }^{4-9}$ However, there have not been many attempts to unify the theoretical study of liquid-mesophase-solid transitions in systems with orientable molecules and to understand carefully how such phases occur via an interplay of the parameters that favor orientational and positional orderings. Lee, Tan, and Woo ${ }^{10}$ and, recently, Frenkel and Mulder," Singh and Singh," and McMullen and Oxtoby ${ }^{13}$ have taken some steps towards such a unification: they have studied transitions between isotropic liquid, nematic, plasticcrystalline, and some orientationally ordered phases. Singh and Singh ${ }^{12}$ and McMullen and Oxtoby ${ }^{13}$ use the Ramakrishnan-Yussouff density-wave theory. ${ }^{4,5}$ We also use this density-wave theory to study the systematics of liquid-mesophase-solid transitions and extend significantly the work of Singh and Singh ${ }^{12}$ and McMullen and Oxtoby. ${ }^{13}$ In particular, we examine transitions between the following phases: isotropic liquid $(L)$; nematic $(N) ;^{1-3,14}$ smectic $(\mathrm{Sm}) ;^{1-3,14,15}$ discotic $(D) ;^{16,17}$ a bcc plastic crystal (bcc $P$ ) with no orientational order; ${ }^{14,18,19}$ two bcc crystals with orientational order $(\mathrm{bccO} 1$ and bccO2), which differ because they have different lattice constants; and an incommensurate bcc crystal with orientational order $(I \mathrm{bcc} O)$. We do not distinguish between smectic- $A$ and smectic-C phases ${ }^{1-3,14,15}$ because these phases have the same free energies given the approximations that we will make (Sec. II).

Extensive experimental work has shown that systems with liquid-crystalline phases exhibit a rich variety of phase diagrams. ${ }^{1-3,14,15}$ Over the past 15 years such phase diagrams have been studied by numerical simulations of orientable molecules of various sorts. ${ }^{11,20-31}$
Some of these simulations use lattice models, so that they are suitable only for the study of orientationally ordered phases. However, other simulations study continuum models with hard ellipses ${ }^{21}$ (in two dimensions) and hard spherocylinders $^{22,30}$ and hard ellipsoids of revolution ${ }^{29}$ (in three dimensions). These continuum models are suitable for the study of both positionally and orientationally ordered phases. Our study has been motivated by a desire to understand the results of the simulations of these continuum models and the experiments on mesogenic systems (e.g., systems with liquid-crystalline mesophases).

There are some obvious limitations of our study. (1) We do not consider various crystalline and liquidcrystalline phases: fcc, hcp, cholesteric, ${ }^{1-3}$ and smectic$B-I, 1-3,15$ to name but a few. (2) We use the Ramakrishnan-Yussouff theory, which neglects fluctuations that are important at continuous transitions (e.g., many nematic-to-smectic- $\boldsymbol{A}$ transitions ${ }^{32}$ ) and weakly first-order transitions (e.g., the liquid-to-nematic transi-

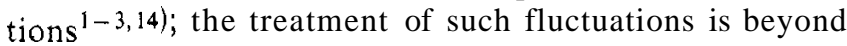
the scope of this paper. Many qualitative features of the phase diagrams we obtain are insensitive to these limitations; some are not. In Sec. V we discuss which of these qualitative features are incorrect, what the correct features are, and how they may be obtained.

Before presenting the details of the calculations, we summarize our principal results. To do so, it is necessary to introduce the elements of the Ramakrishnan-Yussouff theory. ${ }^{4-6}$ This theory is a molecular-field theory ${ }^{3}$ in which the two-particle direct correlation function ${ }^{33}$ plays the role of a two-particle potential (Sec. II). This correlation function, required as an input for the theory, can be obtained, in principle, by measurements in the (supercooled) liquid phase (Sec. II) or from theories of the liquid phase (Sec. IV). In systems of nonspherical molecules the direct correlation function of the liquid $c\left(\mathbf{r}_{12}, \widehat{\mathbf{\Omega}}_{1}, \hat{\mathbf{\Omega}}_{2}\right)$ depends on $\mathbf{r}_{12}=\mathbf{r}_{1}-\mathbf{r}_{2}$, where $\mathbf{r}_{1}$ and $\mathbf{r}_{2}$ specify the positions of the centers of masses of molecules 
1 and 2 , and on $\hat{\boldsymbol{\Omega}}_{1}$ and $\hat{\boldsymbol{\Omega}}_{2}$, which specify the orientations of these molecules, We restrict ourselves to the study of systems with only one type of molecule, which has both cylindrical and center-of-inversion symmetry. For such systems $c\left(\mathbf{r}_{12}, \hat{\mathbf{\Omega}}_{1}, \hat{\mathbf{\Omega}}_{2}\right)$ can be expanded in terms of the spherical harmonics $\boldsymbol{Y}_{l_{1} m_{l}},\left(\hat{\mathbf{\Omega}}_{1}\right), \quad \boldsymbol{Y}_{l_{2} m_{2}}\left(\hat{\mathbf{\Omega}}_{2}\right), \quad$ and $Y_{L M}\left(\hat{\mathbf{r}}_{12}\right)$; a subsequent Fourier transform over $\left|\mathbf{r}_{12}\right|$ yields the generalized Fourier coefficients $\widetilde{c}_{1_{1} l_{2} L}(q)$ of $c\left(\mathbf{r}_{12}, \hat{\mathbf{\Omega}}_{1}, \hat{\mathbf{\Omega}}_{2}\right)$, where $q$ is the magnitude of the wave vector (Sec. II). Symmetry considerations force $1_{1}, l_{2}$, and $\boldsymbol{L}$ to be even integers.

In our study we assume

$$
\overline{\boldsymbol{c}}_{l_{1} l_{2} L}(q)=0 \text { for } L \neq 0 \text { or } l_{1}, l_{2}>2 .
$$

(We examine the consequences of this approximation in Secs. II -V.) Thus the two functions

$$
c_{0}(q) \equiv \frac{\rho_{\mathrm{liq}}}{(4 \pi)^{1 / 2}} \widetilde{c}_{000}(q)
$$

and

$$
c_{2}(q) \equiv \frac{\rho_{\mathrm{liq}}}{(20 \pi)^{1 / 2}} \tilde{c}_{220}(q)
$$

are the only input required for the theory we describe in Sec. II. [We have defined $c_{0}(q)$ and $c_{2}(q)$ with the normalization factors $(4 \pi)^{-1 / 2}$ and $(20 \pi)^{-1 / 2}$ for convenience in the following discussions; $\rho_{\text {liq }}$ is the density of the liquid.]

Different forms of the functions $c_{0}(q)$ and $c_{2}(q)$ lead to different forms of ordering. Figure 1 shows schematic plots of these functions. If the peak in $c_{0}(q)$ at $q=q_{0}$ is sufficiently large, then the free energy of the system is minimized by the formation of a density wave (wave vector of magnitude $q_{0}$ ) with no orientational order; the precise nature of this density wave (bcc, fcc, etc.) depends on other features of the function $c_{0}(q)$, such as its value at secondary maxima. ${ }^{4-6}$ Similarly, the peak in $c_{2}(q)$ at $q=0$ favors the formation of an orientationally ordered (nematic) phase with no positional order; the peak in $c_{2}(q)$ at $q=q_{2}$ favors the formation of density waves (wave vector of magnitude $\simeq q_{2}$ ) with orientational order.

We parametrize the functions $c_{0}(q)$ and $c_{2}(q)$ by their values at $q=0, q=q_{0}$, and $q=q_{2}$. At all other values of $q$ we take these functions to be zero. We also assume that $c_{0}(q=0)=-\infty$, i.e., the liquid is incompressible. (We examine the consequences of these approximations in Secs. II-V.)

We now present various sections through the phase diagram in the five-dimensional space of parameters $c_{0}\left(q_{0}\right)$, $c_{0}\left(q_{2}\right), c_{2}(0), c_{2}\left(q_{0}\right)$, and $c_{2}\left(q_{2}\right)$; we relate them below to phase diagrams in spaces of parameters that can be controlled easily in experiments and numerical simulations.

In the systems we consider, we expect ${ }^{34}$ that $q_{2}<q_{0}$. Thus we see from Fig. 1 that in these systems $c_{0}\left(q_{2}\right)$ is large and negative. The phase diagrams shown in Figs. 2(a)-2(d) are obtained at one fixed value of $c_{0}\left(q_{2}\right)$, which is large and negative, and for different (but fixed) values of $c_{2}\left(q_{0}\right)$. (These are schematic phase diagrams; they are based on the phase diagrams obtained by doing the calcu-

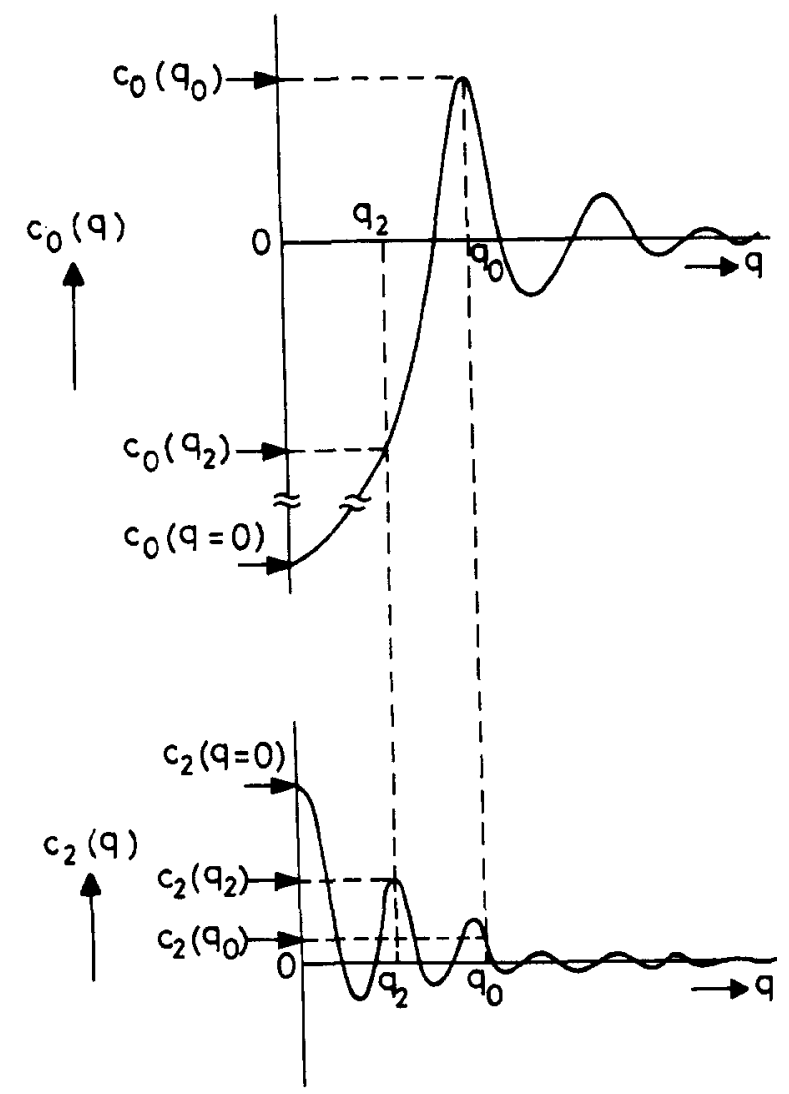

FIG. 1. Schematic plots of direct-correlation-function components $c_{0}(q)$ and $c_{2}(q)$ [for definitions see Eqs. 2(a) and 2(b)] vs $q$ for the isotropic liquid phase of a system of orientable molecules.

lations described in Secs. II and III.) As long as $c_{0}\left(q_{2}\right)<0$, the topologies of the phase diagrams are as shown in these figures. Sum rules force the following inequalities: $c_{0}\left(q_{0}\right) \leq 1$ and $c_{2}\left(q_{0}\right) \leq 1$. Also, in systems of physical interest we expect (Fig. 1) $c_{2}\left(q_{2}\right)<c_{2}(0)$. Thus we plot phase diagrams in the cube $0 \leq c_{0}\left(q_{0}\right) \leq 1$, $0 \leq c_{2}(0) \leq 1$, and $0 \leq c_{2}\left(q_{2}\right) \leq 1$; we expect only the region with $c_{2}\left(q_{2}\right)<c_{2}(0)$ to be of physical interest.

At small and negative values of $c_{2}\left(q_{0}\right)(\Sigma 0)$ we obtain the phase diagram of Fig. 2(a); when $c_{2}\left(q_{0}\right)=0$ we get the phase diagram shown in Fig. 2(b); as we make $c_{2}\left(q_{0}\right)$ more and more positive, we obtain the phase diagrams of Figs. 2(c) and 2(d).

All the phase transitions we obtain are first-order transitions. These occur at first-order phase boundaries. In Figs. 2(a)-2(d), bold lines indicate where phase boundaries meet the faces of the unit cube in which we display our phase diagrams. Lines of three-phase coexistence are shown as dot-dashed lines; lines of four-phase coexistence $^{35}$ are shown as dashed lines. Points of four-, five-, and six-phase coexistence ${ }^{35}$ are indicated, respectively, by squares, pentagons, and closed circles.

As $c_{2}\left(q_{0}\right)$ increases from the value which yields Fig. 2(a) to the one which yields Fig. 2(b), the orientationally ordered bec phase bcc 01 grows at the expense of the plastic-crystalline phase bcc $P$ and the nematic phase $N$. 

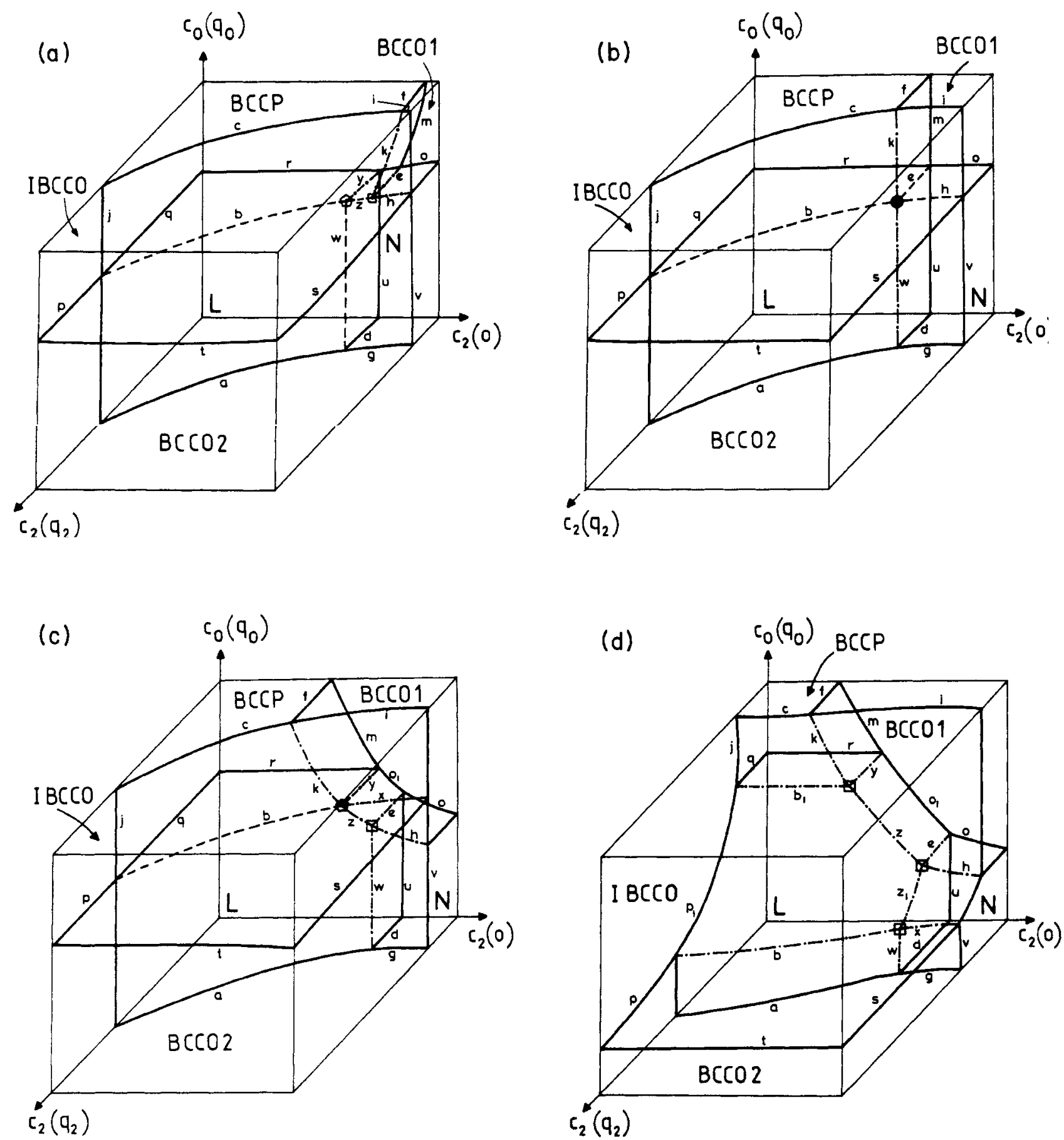

FIG. 2. Three-dimensional sections $\left[c_{2}(0)-c_{0}\left(q_{0}\right)-c_{2}\left(q_{2}\right)\right.$ unit cube] of the phase diagram, for large negative $c_{0}\left(q_{2}\right)$ and varying $c_{2}\left(q_{0}\right)$. The sections are extrapolated from phase diagrams calculated for various two-dimensional sections. The notations for the phases are set down in Table I. The bold solid lines show the intersections of the phase boundaries with the faces of the unit cube. Dashed lines are lines of four-phase coexistence and dot-dashed lines are lines of three-phase coexistence. Open squares, open pentagons, and solid circles represent, respectively, points of four-, five-, and six-phase coexistence (Ref. 35). In terms of the lines identified in these figures, the various phases are bounded by the following surfaces (in addition to the cube faces). (a) $c_{2}\left(q_{0}\right)<0$. (i) $L:(a w b)$, (dwyu), (byrq); (ii) N: (vgwzh), (dwyu), (yze), (heo); (iii) bccO 1; (hki), (heo), (ekfm); (iv) bccO2: (aguxb), (tsxbp); (v) bccP (bzkcj), (yze), (ekfm), (qbyr); (vi) IbccO: (tsxbp), (xbjci). (b) $c_{2}\left(q_{0}\right)=0$. (i) $L:(a w b),(d w e u),(b q r e)$; (ii) $N$ : (gwhv), (dweu), (heo); (iii) bccO 1 : (heo), (ekfm), (hki); (iv) bccO2; (agvhb), (tshbp); (v) bccP: (bjck), (berq), (emfk); (vi) IbccO: (tshbp), (hbjci). (c) c $\left(q_{0}\right)>0$. (i) L: $(a w z b),(d w e u),\left(z e o_{1} y\right),(b y r q)$; (ii) N: (gwhv), (dweu), (heo); (iii) bccO 1: (ikzh), (kfmy), (zyo, $\left.e\right),(h e o)$; (iv) bcco2: (agvxb), (tsxbp); (v) bccP: $(b k c j),(k f m y),(b y r q)$; (vi) IbccO $(t s x b p),(x b j c i)$. (d) Large and positive $c_{2}\left(q_{0}\right)$ (we give only the simplest possible phase diagram). (i) $L$ : (awb), $\left(b z_{1} z b_{1} p_{1}\right),\left(d w z_{1} e u\right),\left(e z y o_{1}\right),\left(b_{1} y r q\right)$; (ii) $N:(g w x v),\left(x z_{1} h\right),(h e o),\left(z_{1} w d u e\right)$; (iii) bccol: (ikzh), (ykfm), $\left(z y o_{1} e\right)$, (heo); (iv) bccO2: (agvxb), (tsxbp); (v) bccP $\left(b_{1} k c j\right),\left(b_{1} y r q\right),(y k f m)$; (vi) IbccO: $(t s x b p),\left(x z_{1} h\right),\left(b z_{1} z b_{1} p_{1}\right),\left(b_{1} k c j\right)$, $(i k z h)$. 
As $c_{2}\left(q_{0}\right)$ increases further [Figs. 2(c) and 2(d)], bccO 1 continues this growth, now also at the expense of the liquid $\boldsymbol{L}$. Furthermore, the incommensurate, orientationally ordered phase $\mathrm{IbccO}$ also starts growing slowly, at the expense of the liquid $L$, the bce plastic crystal $b c c P$, and the second orientationally ordered bcc crystal bcc O2; however, this growth is appreciable only when $c_{2}\left(q_{0}\right)$ becomes greater than $K_{1}$ [a positive number which depends on the value of $\left.c_{0}\left(q_{2}\right)\right]$. The growth of the phase bccO 1 continues as $c_{2}\left(q_{0}\right)$ increases, and eventually no lines (points) of four-phase (five-phase) coexistence remain [as in Fig. 2(d)] when $\boldsymbol{c}_{2}\left(q_{0}\right)>K_{2}$ [whose value depends on $\left.c_{0}\left(q_{2}\right)\right]$.

The most significant result of our study is that neither smectic nor discotic phases appear in the phase diagrams of Figs. 2(a) $-2(d)$. This is a surprising but important result because various molecular-field theories of smectic $^{\prime-3,10,36-39}$ and discotic ${ }^{40}$ orderings are special cases of the theory we use. (We show this in detail in Sec. II.) The reason for the discrepancy between our theory and these molecular-field theories is that the latter do not allow for three-dimensional positional ordering $(b c c$, in particular) at the same wave vector $q_{2}$ (Fig. 1) at which they allow for smectic or discotic ordering. ${ }^{10,36-40}$ (This shortcoming of such molecular-field theories has been pointed out, in a slightly different context, by Harrowell and Oxtoby. ${ }^{41}$ ) Smectic and discotic phases appear only as metastable phases in our theory, i.e., they correspond to local but not global minima of the variational free energy we use (Secs. II and III); the oriented boc crystal (bccO2) always has a lower free energy than the smectic and discotic phases in our theory. Roughly speaking, the reason for this is as follows: smectic, discotic, and becO2 phases appear when the system lowers its free energy by creating oriented density waves with wave vector $q_{2}$. Since there are 2 such waves in a smectic phase, 6 in a discotic phase," and 12 in a bccO2 phase, the system lowers its free energy the most by forming a bcc 02 phase (i.e., $F_{\mathrm{Sm}}>F_{D}>F_{\mathrm{bcc} O 2}$, where $\boldsymbol{F}$ stands for the free energy and the subscripts refer to the phases); the bcc phase is further stabilized by cubic terms in a Landau expansion of our free energy. The molecular-field theories ${ }^{10,36-40}$ use $c_{0}\left(q_{2}\right)=0$. Even at this value of $c_{0}\left(q_{2}\right)$ we do not find thermodynamically stable smectic or discotic phases (Sec. 111). Nor do we find such stable smectics or discotics at negative values of $c_{0}\left(q_{2}\right)$ which disfavor the formation of the bccO2 phase more than they disfavor the formation of discotic and smectic phases. At large and negative values of $c_{0}\left(q_{2}\right)$ the free energies of the metastable smectic and discotic phases come close to the free energy of the stable bcc 22 phase, but the latter still has the lowest free energy (Sec. III).

For mesogenic systems, experimental phase diagrams are available mostly along the temperature $(T)$ axis $^{1,2,14,15}$ or, in some cases, in the pressure-temperature $(P-T)$ plane. ${ }^{42}$ Smectics and discotics are commonly found in such phase diagrams; however, they are embarrassingly absent in the phase diagrams of Fig. 2. [As we will discuss in detail in Sec. V, we believe that we need to relax some of the constraints imposed in Eq. (1) to obtain smectic and discotic phases.] Nevertheless, it is interest- ing to map the phase diagrams of Fig. 2 onto P-T phase diagrams to see which experimental features our theory can account for. This mapping is not easy: to convert the phase diagrams of Fig. 2 into $\boldsymbol{P} \cdot \boldsymbol{T}$ phase diagrams, we need to know how the direct correlation function depends on $\mathrm{P}$ and $\boldsymbol{T}$. However, to the best of our knowledge, even the two functions $c_{0}(q)$ and $c_{2}(q)$ [let alone $\left.c_{l_{1} l_{2} L}(q)\right]$ are not known for any mesogenic system at any temperature or pressure.

Nevertheless, we can use the following rough arguments to show that there is a qualitative correspondence between the phase diagrams of Fig. 2 and experimental phase diagrams: as the temperature $\boldsymbol{T}$ decreases, the peaks of $\boldsymbol{c}_{0}(q)$ and $\boldsymbol{c}_{2}(q)$ rise. Thus, lowering the temperature is equivalent to following a path that moves radially outward from the origin in the phase diagrams of Fig. 2. Therefore the sequence that might result upon cooling a mesogenic system can be obtained by following radial paths (not straight in general) outward from the origins of Figs. 2(a)-2(d). Note, in particular, that it is possible to obtain the sequence $\boldsymbol{L} \rightarrow \boldsymbol{N} \rightarrow \mathrm{bcc} P \rightarrow$ bccO 1 , as suggested by recent experiments; ${ }^{43}$ however, given the topologies of Figs. 2(a) -2(d), such a sequence of transitions is improbable and should occur, at best, only in a few mesogenic systems.

Figure $\mathbf{3}$ shows a plot of the phase diagram of a system of hard ellipsoids of revolution. This phase diagram is based on the numerical simulations of Frenkel, Mulder, and McTague. ${ }^{11,29}$ We plot it in the packing fraction $(\eta)$-eccentricity $(a / b)$ plane. ( $a$ is one half the height of

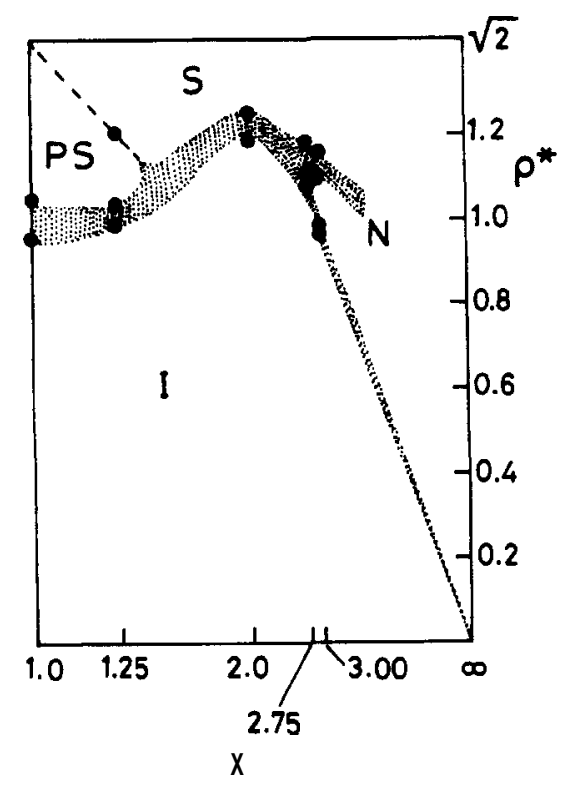

FIG. 3. A part of the phase diagram for a system of hard ellipsoids of revolution as obtained from the molecular-dynamics simulation of Frenkel and Mulder (Ref.11). The symbols $I, N$, PS, and $S$ represent, respectively, isotropic liquid, nematic, fcc plastic crystal, and (distorted) fcc crystalline phases. $\rho^{*}$ is the reduced density related to the packing fraction $\eta$ through $\eta=(\pi / 6) \rho^{*}$. Shaded areas are regions of two-phase coexistence. 
the axis of revolution and $b$ is one half the width of the ellipsoids.) To compare the phase diagrams of Figs. 2 and 3 , we must know how the direct correlation function $c\left(\mathbf{r}_{12}, \hat{\boldsymbol{\Omega}}_{1}, \widehat{\boldsymbol{\Omega}}_{2}\right)$ varies as a function of $\boldsymbol{\eta}$ and $a / b$ for a system of hard ellipsoids of revolution. This is not known exactly. In Sec. IV we use an approximation for $\boldsymbol{c}\left(\mathbf{r}_{12}, \hat{\mathbf{\Omega}}_{1}, \hat{\mathbf{\Omega}}_{2}\right)$ of a system of hard ellipsoids of revolution to compare the phase diagrams of Figs. 2 and 3. We obtain a fair, qualitative agreement between these phase diagrams for the liquid-to-plastic crystal and liquid-tonematic transitions, but not for other transitions. This is not surprising because the approximation we use for the direct correlation function is reasonable only in the liquid phase at a low packing fraction $\eta$ (Sec. IV). Another qualitative feature common to the phase diagrams of Figs. 2 and 3 is the absence of smectic and discotic phases. (See Secs. IV and V for a detailed discussion of this point.) There are, of course, qualitative differences between Figs. 2 and 3: since our theory only allows for bcc positional ordering, it does not yield the change in crystal structure obtained in going from the plasticcrystalline phase to the orientationally ordered phase in Fig. 3. Also, in the numerical simulations that yield Fig. 3 , the change in the orientational order parameter is continuous across the phase boundary that separates the plastic-crystal phase from the orientationally ordered crystalline phase; in our calculations the change in this order parameter is discontinuous across this phase boundary. Finally, the reason that our calculations do not yield any discontinuous density changes at phase boundaries, unlike the numerical simulations, ${ }^{11,29}$ is because we make the approximation that the fluid phase is incompressible.

Recent simulations ${ }^{30}$ of a system of parallel hard spherocylinders have obtained a smectic phase in such systems. It is not clear to us why a system of parallel hard spherocylinders shows a smectic phase, whereas a system of hard ellipsoids of revolution does not. We comment on this point further in Secs. IV and V.

The remaining part of this paper is organized as follows. In Sec. II we describe the Ramakrishnan-Yussouff theory and discuss how it has to be extended to study liquid-mesophase-solid transitions. In Sec. III we present the numerical results that lead to phase diagrams like those shown in Fig. 2. In Sec. IV we compare our results with experiments and numerical simulations. In Sec. $\mathrm{V}$ we conclude this paper with a discussion of the shortcomings of our theory and how they may be overcome.

\section{DENSITY-WAVE THEORY FOR ORDERING IN LIQUID-CRYSTALLINE SYSTEMS}

The Ramakrishnan-Yussouff density-wave theory for the freezing of a liquid has been described in various places. $^{4-9}$ Its extension to the study of liquid-crystalline phases is straightforward and has been discussed by several authors. ${ }^{44-46}$ To make this paper self-contained, we give a brief outline of this density-wave theory.

The basis for the Ramakrishnan-Yussouff density-wave theory for the ordering of liquids is an expansion for the free-energy functional $\mathrm{F}$ for a nonuniform density $\rho(r, \widehat{\Omega})$ (the average density of molecules at the point $r$ with orientation $\widehat{\Omega}$ ),

$$
\begin{aligned}
\beta\left(F-F_{\text {liq }}\right)= & \beta \int d \mathbf{r} \int d \hat{\mathbf{\Omega}} \rho(\mathbf{r}, \hat{\mathbf{\Omega}}) v_{e}(\mathbf{r}, \hat{\mathbf{\Omega}})+\int d \mathbf{r} \int d \hat{\mathbf{\Omega}}\left\{\rho(\mathbf{r}, \hat{\mathbf{\Omega}}) \ln \left[\rho(\mathbf{r}, \hat{\mathbf{\Omega}}) / \rho_{\mathrm{liq}}\right]-\left[\rho(\mathbf{r}, \hat{\mathbf{\Omega}})-\rho_{\mathrm{liq}}\right]\right\} \\
& -\frac{1}{2 !} \int d \mathbf{r}_{1} \int d \hat{\mathbf{\Omega}}_{1} \int d \mathbf{r}_{2} \int d \hat{\mathbf{\Omega}}_{2} c^{(2)}\left(\mathbf{r}_{1}, \mathbf{r}_{2}, \hat{\mathbf{\Omega}}_{1}, \hat{\mathbf{\Omega}}_{2}\right)\left[\rho\left(\mathbf{r}_{1}, \hat{\mathbf{\Omega}}_{1}\right)-\rho_{\mathrm{liq}}\right]\left[\rho\left(\mathbf{r}_{2}, \hat{\mathbf{\Omega}}_{2}\right)-\rho_{\mathrm{liq}}\right] \\
& -\frac{1}{3 !} \int d \mathbf{r}_{1} \int d \hat{\mathbf{\Omega}}_{1} \int d \mathbf{r}_{2} \int d \hat{\mathbf{\Omega}}_{2} \int d \mathbf{r}_{3} \int d \hat{\mathbf{\Omega}}_{3} c^{(3)}\left(\mathbf{r}_{1}, \mathbf{r}_{2}, \mathbf{r}_{3}, \hat{\mathbf{\Omega}}_{1}, \hat{\mathbf{\Omega}}_{2}, \hat{\mathbf{\Omega}}_{3}\right)\left[\rho\left(\mathbf{r}_{1}, \hat{\mathbf{\Omega}}_{1}\right)-\rho_{\mathrm{liq}}\right] \\
& \times\left[\rho\left(\mathbf{r}_{2}, \hat{\mathbf{\Omega}}_{2}\right)-\rho_{\mathrm{liq}}\right]\left[\rho\left(\mathbf{r}_{3}, \hat{\mathbf{\Omega}}_{3}\right)-\rho_{\mathrm{liq}}\right]-\cdots
\end{aligned}
$$

Here $F_{\text {liq }}$ is the free energy of the uniform, isotropic liquid (supercooled) of density $\rho_{\text {liq }} ; \beta=1 /\left(k_{B} T\right) ; k_{B}$ is the Boltzmann constant; $v_{e}(\mathbf{r}, \hat{\boldsymbol{\Omega}})$ is an external, one-body potential; and $c^{(2)}, c^{(3)}$, etc., are the direct correlation functions in the isotropic liquid, which are given by

$$
\begin{aligned}
c^{(2)}\left(\mathbf{r}_{1}, \mathbf{r}_{2}, \hat{\mathbf{\Omega}}_{1}, \hat{\mathbf{\Omega}}_{2}\right)= & \left.\frac{-\delta^{2}\left[\beta\left(F-F_{\mathrm{liq}}\right)\right]}{\delta \rho\left(\mathbf{r}_{1}, \hat{\mathbf{\Omega}}_{1}\right) \delta \rho\left(\mathbf{r}_{2}, \hat{\mathbf{\Omega}}_{2}\right)}\right|_{\rho_{\text {liq }}} \\
& +\frac{\delta\left(\mathbf{r}_{1}-\mathbf{r}_{2}\right) \delta\left(\hat{\mathbf{\Omega}}_{1}-\hat{\mathbf{\Omega}}_{2}\right)}{\rho_{\text {liq }}},
\end{aligned}
$$

etc. Translational invariance yields $c^{(2)}\left(\mathbf{r}_{1}, \mathbf{r}_{2}, \hat{\mathbf{\Omega}}_{1}, \hat{\mathbf{\Omega}}_{2}\right)$ $=c^{(2)}\left(\mathbf{r}_{12}, \widehat{\mathbf{\Omega}}_{1}, \widehat{\mathbf{\Omega}}_{2}\right)$, where $\mathbf{r}_{12}=\mathbf{r}_{1}-\mathbf{r}_{2}$.

If we minimize $\beta\left(\boldsymbol{F}-F_{\text {liq }}\right)$ with respect to $\rho(\mathbf{r}, \hat{\boldsymbol{\Omega}})$, we get

$$
\frac{\rho(\mathbf{r}, \hat{\mathbf{\Omega}})}{\rho_{\text {liq }}}=\exp \left[-\beta v_{\text {eff }}(\mathbf{r}, \hat{\mathbf{\Omega}})\right]
$$

where

$$
\begin{aligned}
-\beta v_{\mathrm{eff}}(\mathbf{r}, \hat{\mathbf{\Omega}})= & -\beta v_{e}(\mathbf{r}, \hat{\mathbf{\Omega}})+\int d \mathbf{r}_{1} \int d \hat{\mathbf{\Omega}}_{1} c^{(2)}\left(\mathbf{r}, \mathbf{r}_{1}, \hat{\mathbf{\Omega}}, \hat{\mathbf{\Omega}}_{1}\right)\left[\rho\left(\mathbf{r}_{1}, \hat{\mathbf{\Omega}}_{1}\right)-\rho_{\text {liq }}\right] \\
& +\frac{1}{2} \int d \mathbf{r}_{1} \int d \hat{\mathbf{\Omega}}_{1} \int d \mathbf{r}_{2} \int d \hat{\mathbf{\Omega}}_{2} c^{(3)}\left(\mathbf{r}, \mathbf{r}_{1}, \mathbf{r}_{2}, \hat{\mathbf{\Omega}}, \hat{\mathbf{\Omega}}_{1}, \hat{\mathbf{\Omega}}_{2}\right)\left[\rho\left(\mathbf{r}_{1}, \hat{\mathbf{\Omega}}_{1}\right)-\rho_{\text {liq }}\right]\left[\rho\left(\mathbf{r}_{2}, \hat{\mathbf{\Omega}}_{2}\right)-\rho_{\text {liq }}\right]+\cdots,
\end{aligned}
$$


i.e., $\rho(\mathbf{r}, \widehat{\mathbf{\Omega}})$ essentially assumes the value it would have in a noninteracting fluid with an effective, one-body potential (molecular-field), which is self-consistently determined by Eqs. (5) and (6). [Since Ramakrishnan and Yussouff $f^{4}$ do not consider the possibility of orientational ordering, they have $\rho(\mathbf{r}, \widehat{\boldsymbol{\Omega}})=\rho(\boldsymbol{r})$ and $c^{(2)}\left(\mathbf{r}_{12}, \widehat{\boldsymbol{\Omega}}_{1}, \widehat{\boldsymbol{\Omega}}_{2}\right)$ $=c^{(2)}\left(\left|r_{1}-r_{2}\right|\right)$, etc.] In the remainder of this paper we neglect in Eqs. (3) and (6) the terms containing the correlation functions $c^{(n)}$, with $n \geq 3$.

Note that if we assume that the particles of the fluid interact via a pair potential $\boldsymbol{V}^{(2)}\left(\mathbf{r}_{12}, \widehat{\mathbf{\Omega}}_{1}, \widehat{\mathbf{\Omega}}_{2}\right)$, then molecular-field approximations ${ }^{3,36-40}$ use an effective, one-body potential

$$
\int d \mathbf{r}_{1} d \hat{\mathbf{\Omega}}_{1} V^{(2)}\left(\left(\mathbf{r}-\mathbf{r}_{1}\right), 8, \hat{\mathbf{\Omega}}_{1}\right)\left[\rho\left(\mathbf{r}_{1}, \hat{\mathbf{\Omega}}_{1}\right)-\rho_{\mathrm{liq}}\right]
$$

in addition to any externally imposed potential. Thus these molecular-field approximations are special cases of the Ramakrishnan-Yussouff approximation ${ }^{47}$ with a $c^{(2)}$ that is assumed to be $-\beta V^{(2)}$.

Our task is now clear: we have to find solutions $\rho(\mathbf{r}, \hat{\boldsymbol{\Omega}})$ of Eqs. (5) and (6) with $v_{e}$ set equal to 0 . If there is more than one solution, we pick the one which yields the lowest minimum of the functional $\boldsymbol{F}-F_{\text {liq }}[\boldsymbol{E} \boldsymbol{q}$. (3)]; this solution yields the density distribution in the thermodynamically stable phase.

Equations (5) and (6) (with $v_{e}=0$ ) always have the trivial solution $\rho(\mathbf{r}, \hat{\mathbf{\Omega}})=\rho_{\text {liq }}$, i.e., the isotropic liquid with a uniform density $\rho_{\text {liq }}$, To investigate the existence of nontrivial solutions, in particular, solutions which characterize phases with orientational or positional ordering (or both), it is convenient to expand $\rho(\mathbf{r}, \hat{\mathbf{\Omega}})$ as

$$
\rho(\mathbf{r}, \hat{\boldsymbol{\Omega}})=\rho_{\text {liq }}\left[1+\sum_{\boldsymbol{G}} \sum_{i, m} e^{i \mathbf{G} \cdot \mathbf{r}} \mu_{l m}(G) Y_{l m}(\hat{\mathbf{\Omega}})\right] .
$$

The expansion coefficients $\mu_{I m}(G)$ are order parameters that characterize different types of positional and orientational ordering [e.g., in a phase with only positional or$\operatorname{der}^{4,5} \mu_{l m}(G)=0$, for $l, m \neq 0$, and $\mu_{00}(G) \neq 0$; in a phase with only orientational order, ${ }^{48} \mu_{I m}(G)=0$, for $G \neq 0$, and $\mu_{l m}(0) \neq 0$ ]. Note that the nature of the positional ordering depends not only on the order parameters but also on the set of reciprocal-lattice vectors $G$ used in Eq. (7).

We can also expand $c^{(2)}\left(\mathbf{r}_{12}, \widehat{\mathbf{\Omega}}_{1}, \hat{\mathbf{\Omega}}_{2}\right)$ [henceforth we drop the superscript (2)]. ${ }^{49}$

$$
\begin{aligned}
c\left(\mathbf{r}_{12}, \hat{\mathbf{\Omega}}_{1}, \hat{\mathbf{\Omega}}_{2}\right)=\sum_{\substack{l_{1}, l_{2}, L, m_{1}, m_{2}, M}} & {\left[\begin{array}{ccc}
l_{1} & l_{2} & L \\
m_{1} & m_{2} & M
\end{array}\right] Y_{l_{1} m_{1}}\left(\hat{\mathbf{\Omega}}_{1}\right) } \\
& x Y_{l_{2} m_{2}}\left(\hat{\mathbf{\Omega}}_{2}\right) Y_{L M}\left(\hat{\mathbf{r}}_{12}\right) \\
& \times c_{l_{1} l_{2} L}\left(r_{12}\right),
\end{aligned}
$$

where $\left(\begin{array}{ccc}l_{1} & l_{2} & L \\ m_{1} & m_{2} & M\end{array}\right)$ is a Wigner $3-j$ symbol,${ }^{50}$ the $Y_{\mathrm{lm}}$ 's are spherical harmonics, $r_{12}=\left|\mathbf{r}_{12}\right|$, and $\widehat{\mathbf{r}}_{12}=\mathbf{r}_{12} / r_{12}$. If we define

$$
\widetilde{c}_{l_{1} l_{2} L}(G)=\int_{0}^{\infty} d r(i)^{L}(2 L+1) j_{L}(G r) c_{l_{1} l_{2} L}(r),
$$

where the $j_{L}$ 's are spherical Bessel functions, then by sub- stituting Eqs. (7) - (9) into Eqs. (5) and (6) (with $c^{(n)}=0$ for $n \geq 3$ ) we get an infinite set of coupled equations for the order parameters $\mu_{l m}(G)$. We now make the following approximations.

(1) We restrict our study to systems with molecules of only one type. Thus we need to consider only even values of $\boldsymbol{L}$ in Eqs. (8) and (9) [i.e., $c_{l_{1} l_{2} L}\left(r_{12}\right)=0$ for odd $L$ ]. ${ }^{49}$

(2) We restrict our study to systems with molecules which have both cylindrical and center-of-inversion symmetry. Thus we need to consider only even values of $I$, and $l_{2}$ in Eqs. (8) and (9) and even values of $I$ in Eq. (7). ${ }^{49}$

(3) We assume that $\widetilde{\boldsymbol{c}}_{l_{1} l_{2} L}(G)=0$ for $L \neq 0$ [Eq. (1)]; i.e., we assume that the interaction between two molecules 1 and 2 depends upon their orientations $\hat{\boldsymbol{\Omega}}_{1}$ and $\hat{\boldsymbol{\Omega}}_{2}$ and upon the distance $r_{12}$ between their centers, but not on $\widehat{\mathbf{r}}_{12}$. Since $\boldsymbol{L}=0$ is the only value of $L$ we allow in Eqs. (8) and (9), we have $l_{1}=l_{2} \equiv l$ and $m_{1}=-m_{2} \equiv m$. By choosing the direction along which the molecules align in orientationally ordered phases to be the $z$ axis, we can take $\boldsymbol{m}=0$. (One of the consequences of this approximation is that smectic-A and smectic-C phases have the same free energies in our theory.)

(4) We further assume that $\widetilde{\boldsymbol{c}}_{l_{1} l_{2} L}(G)=0$ for $I, l_{2}>2$ [Eq. (1)]. this assumption is based on the hope that loworder (i.e., low $l_{1}, l_{2}$, and $L$ ) $\overline{\boldsymbol{c}}_{l_{1} l_{2} L}(G)$ 's suffice to stabilize the phases we are interested in (Sec. I).

(5) We parametrize the functions $\tilde{\boldsymbol{c}}_{000}(G)$ and $\tilde{\boldsymbol{c}}_{220}(G)$ (or the related functions $c_{0}(G)$ and $c_{2}(G)$ [Eq. (2)]) in terms of their values at $\boldsymbol{G}=0, q_{0}$, and $q_{2}$. (See Fig. 1 and the accompanying discussion for the physical motivation for this approximation.) At all other values of $\boldsymbol{G}$ we assume that $\tilde{\boldsymbol{c}}_{000}(\boldsymbol{G})$ and $\tilde{\boldsymbol{c}}_{220}(\boldsymbol{G})$ are zero.

(6) Finally, we assume that the liquid is incompressible, so that $\widetilde{\boldsymbol{c}}_{000}(0)=-\infty$.

If we make the approximations listed in the preceding paragraphs, the infinite set of equations for the order parameters $\mu_{l m}(G)$ is replaced by a finite set. To write this set of equations it is convenient to define

$$
\begin{aligned}
& \tau_{l}(G) \equiv \frac{(2 l+1)^{1 / 2}}{4 \pi} \mu_{l 0}(G), \\
& c_{l}(G) \equiv \frac{\rho_{\mathrm{liq}}}{(4 \pi)^{1 / 2}}\left(\begin{array}{lll}
l & l & 0 \\
0 & 0 & 0
\end{array}\right) \widetilde{c}_{l l 0}(G), \\
& \widetilde{P}_{l}(\hat{\mathbf{\Omega}} \cdot \hat{\mathbf{z}}) \equiv(2 l+1)^{1 / 2} P_{l}(\hat{\mathbf{\Omega}} \cdot \hat{\mathbf{z}}),
\end{aligned}
$$

and

$$
\phi_{0} \equiv \int d \mathbf{r} \int d \hat{\mathbf{\Omega}} \exp \left(\sum_{l, \mathbf{G}} e^{i \mathbf{G} \cdot \mathbf{r}} \tau_{l}(G) c_{l}(G) \widetilde{P}_{l}(\hat{\mathbf{\Omega}} \cdot \hat{\mathbf{z}})\right],
$$

where the $P_{1}$ 's are Legendre polynomials. Then, given the approximations (1)-(3) and (6), we can rewrite the free energy $(3)$ as $\left(c^{(n)}=0\right.$ for $\left.n \geq 3\right)$

$$
\frac{\beta\left(F-F_{\mathrm{liq}}\right)}{\rho_{\mathrm{liq}} V}=\frac{1}{2} \sum_{\mathrm{G}, l} c_{l}(G)\left[\tau_{l}(G)\right]^{2}-\ln \left(\phi_{0}\right)
$$

and the self-consistent equation for the order parameters as 
TABLE I. Notations for the various mesophases considered in the paper and the molecular fields that describe the phases. [The components $\xi_{0}\left(q_{2}\right)$ and $\xi_{2}\left(q_{0}\right)$ will also be present in the molecular field when $c_{0}\left(q_{2}\right)$ and $c_{2}\left(q_{0}\right)$ are nonzero. But they are rather small, and are not included in the table for the sake of simplicity.]

\begin{tabular}{|c|c|c|}
\hline Notation & Phase & $\begin{array}{c}\text { Molecular field } \\
\xi(\mathbf{r}, \widehat{\Omega})=\ln \left[\rho(\mathbf{r}, \widehat{\Omega}) / \rho_{\text {liq }}\right]\end{array}$ \\
\hline$L$ & Isotropic liquid & 0 \\
\hline$N$ & Nematic phase oriented along $\hat{\mathbf{n}}$ & $\xi_{0}(0)+\xi_{2}(0) P_{2}(\hat{\mathbf{\Omega}} \cdot \hat{\mathbf{n}})$ \\
\hline $\mathrm{Sm}$ & $\begin{array}{l}\text { Smectic phase oriented along } \widehat{\mathrm{n}} \text {, } \\
\text { with layers perpendicular to } \hat{\mathbf{z}}\end{array}$ & $\xi_{0}(0)+\left[\xi_{2}(0)+2 \xi_{2}\left(q_{2}\right) \cos \left(q_{2} \widehat{\mathbf{z}} \cdot \mathbf{r}\right)\right] P_{2}(\hat{\mathbf{\Omega}} \cdot \hat{\mathbf{n}})$ \\
\hline$D$ & $\begin{array}{l}\text { Discotic phase oriented along } \\
6 \text {, with } 2 \text {-d triangular ordering } \\
\text { perpendicular to } \hat{z}\end{array}$ & $\begin{array}{l}\xi_{0}(0)+\xi_{2}(0)+\xi_{2}\left(q_{2}\right) \sum e^{i q_{2} G \cdot \mathbf{r}}-P_{2}(\hat{\mathbf{\Omega}} \cdot \hat{\mathbf{n}}) ; \\
\mathbf{6}: \text { the } 6 \text { smallest (unit } \\
\text { length) reciprocal vectors for } \\
\text { the } 2 \text {-d triangular lattice }\end{array}$ \\
\hline bcc $P$ & $\begin{array}{l}\text { bcc plastic crystal (smallest re- } \\
\text { ciprocal vectors of length } q_{0} \text { ) }\end{array}$ & $\begin{array}{l}\xi_{0}(0)+\xi_{0}\left(q_{0}\right) \sum_{\hat{\boldsymbol{G}}} e^{i q_{0} \hat{\mathrm{G}} \cdot \mathrm{r}} ; \\
\hat{\mathrm{G}} \text { : The } 12 \text { smallest (unit } \\
\text { length) reciprocal vectors for } \\
\text { the bcc lattice }\end{array}$ \\
\hline $\operatorname{bcc} O 1$ & $\begin{array}{l}\text { Oriented bcc crystal (smallest } \\
\text { reciprocal vectors of length } q_{0} \text { ) }\end{array}$ & $\begin{array}{l}\xi_{0}(0)+\xi_{0}\left(q_{0}\right) \sum_{\hat{\sigma}} e^{i q_{0} \hat{\mathbf{G}} \cdot \mathrm{r}}+\xi_{2}(0) P_{2}(\hat{\mathbf{\Omega}} \cdot \hat{\mathbf{n}}) \\
\quad \hat{\mathbf{G}} \text { as in bcc } P\end{array}$ \\
\hline $\mathrm{bccO} 2$ & $\begin{array}{l}\text { Oriented bcc crystal (smallest } \\
\text { reciprocal vectors of length } q_{2} \text { ) }\end{array}$ & $\begin{array}{l}\xi_{0}(0)+\xi_{2}(0)+\xi_{2}\left(q_{2}\right) \sum_{\hat{\boldsymbol{G}}} e^{i q_{2} \hat{\mathrm{O}} \cdot \mathbf{r}} P_{2}(\hat{\boldsymbol{\Omega}} \cdot \hat{\mathbf{n}}) \\
\quad \widehat{\mathrm{G}} \text { as in bccP }\end{array}$ \\
\hline $\mathrm{IbccO}$ & $\begin{array}{l}\text { Incommensurate bcc crystal } \\
\text { with oriented density waves of } \\
\text { wave vectors } q_{2} \text { and (mainly) } \\
\text { unoriented density waves of } \\
\text { wave vector of length } q_{0}\end{array}$ & $\begin{array}{l}\xi_{0}(0)+\left[\xi_{2}(0)+\xi_{2}\left(q_{2}\right) \sum_{\hat{\mathrm{G}}} e^{i q_{2} \hat{\mathrm{G}} \cdot \mathrm{r}}\right] P_{2}(\hat{\mathbf{\Omega}} \cdot \hat{\mathbf{n}}) \\
+\xi_{0}\left(q_{0}\right) e^{i q_{0} \hat{\mathbf{G}} \cdot \mathrm{r}} ; \\
\hat{\mathrm{G}} \text { as in bcc } P\end{array}$ \\
\hline
\end{tabular}

$$
\tau_{l}(G)=\frac{1}{Z_{G} c_{l}(G)} \frac{1}{\phi_{0}} \frac{\partial \phi_{0}}{\partial \tau_{l}(G)}
$$

where $Z_{G}$ is the number of vectors $G$, with $|\boldsymbol{G}|=G$ $\left(Z_{G}=12\right.$ for the first set of reciprocal-lattice vectors for a bcc crystal).

Note that Eqs. (11) and (12) are valid for all $l$ and $G$ given assumptions (1) -(3) and (6) in the preceding paragraphs, i.e., as long as $L=0$ the liquid is incompressible and consists of identical molecules with cylindrical and center-of-inversion symmetry. Assumptions (4) and (5) reduce $\mathrm{Eq}$. (12)to a finite set of equations, since we allow only $1=0$ and 2 and $\mathrm{G}=0, q_{0}$, and $q_{2}$ (Fig. 1 ).

Note further that Eqs. (11) and (12) are different for different types of ordering. The types of ordering (i.e., phases) that we consider are summarized in Table I. The molecular field

$$
\xi(\mathbf{r}, \hat{\mathbf{\Omega}})=\sum_{\mathrm{G}} \xi_{l}(G) e^{i \mathrm{G} \cdot \mathbf{r}} P_{l}(\hat{\mathbf{\Omega}} \cdot \hat{\mathbf{n}})
$$

where $\xi_{l}(G)=\tau_{l}(G) c_{l}(G)$ is also given for each one of these phases.
As we mentioned in Sec. I, various molecular-field theories of liquid-crystalline ordering ${ }^{3,36-40}$ are special cases of the theory we describe here. In these molecularfield theories $c\left(\mathbf{r}_{12}, \widehat{\Omega}_{1}, \widehat{\Omega}_{2}\right)$ is replaced by $-\beta V^{(2)}\left(\mathbf{r}_{12}, \hat{\boldsymbol{\Omega}}_{1}, \hat{\mathbf{\Omega}}_{2}\right)$, where $V^{(2)}$ is a two-body potential. In other words, if instead of trying to determine $c\left(\boldsymbol{r}_{12}, \hat{\boldsymbol{\Omega}}_{1}, \hat{\boldsymbol{\Omega}}_{2}\right)$ from experiments or theories of liquids, we use the approximation

$$
c\left(\mathbf{r}_{12}, \hat{\mathbf{\Omega}}_{1}, \hat{\mathbf{\Omega}}_{2}\right)=-\beta V^{(2)}\left(\mathbf{r}_{12}, \hat{\mathbf{\Omega}}_{1}, \hat{\mathbf{\Omega}}_{2}\right)
$$

then the results of our theory and these molecular-field theories should be the same. Note that these theories make the same approximations that we make ( $L=0$, $l=0,2$, etc.) even though the approximations are not stated as explicitly as we have done. In particular, the theory of McMillan ${ }^{37}$ corresponds to the following choice of parameters:

$c_{2}(0)=\frac{1}{5} \frac{V_{0}}{k_{B} T}, \quad c_{2}\left(q_{2}\right)=\frac{\alpha}{10} \frac{V_{0}}{k_{B} T}, \quad c_{0}\left(q_{0}\right)=\frac{\alpha \delta}{2} \frac{V_{0}}{k_{B} T}$,
$c_{0}\left(q_{2}\right)=0, \quad c_{2}\left(q_{0}\right)=0$

(The parameters $a, \delta$, and $V_{0}$ are as defined in McMillan's work. ${ }^{37}$ ) 


\section{NUMERICAL METHODS AND RESULTS}

Given the approximations that we have made, the infinite set of equations (12) reduces to a set of five, coupled, nonlinear equations in the variables $\tau_{0}\left(q_{0}\right), \tau_{0}\left(q_{2}\right)$, $\tau_{2}(0), \tau_{2}\left(q_{0}\right)$, and $\tau_{2}\left(q_{2}\right)$. We solve these equations by standard iteration or Newton methods.

The evaluation of the right-hand side of Eq. (12) requires some care. In the expression for $\$$ [Eq. (10d)] we expand

$$
\exp \left(\sum_{l, \mathrm{G}} e^{i \mathrm{G} \cdot \mathbf{r}} \tau_{l}(\boldsymbol{G}) \boldsymbol{c}_{l}(\boldsymbol{G}) \widetilde{P}_{l}(\hat{\mathbf{\Omega}} \cdot \hat{\mathbf{z}})\right)
$$

in powers of $\left(1 / \boldsymbol{Z}_{G}\right) \Sigma_{\mathbf{G}} e^{i \mathbf{G} \cdot \mathbf{r}}$ and $\widetilde{P}_{l}(\hat{\mathbf{\Omega}} \cdot \hat{\mathbf{z}})$. We then integrate each term in the series separately. We evaluate the angular integrals analytically and the spatial integrals (over a unit cell of the ordered phase being considered) by using a 24-point Gaussian quadrature. For certain values of $c_{l}(G)$, the resulting series converges slowly, so we retain many terms in the series (up to 85 in some cases) and, to avoid roundoff errors, we use high-precision arithmetic (up to 32 figures in some parts of the calculation and never lower than 16 figures).

In principle, we can obtain the phase diagram in the five-dimensional parameter space of $c_{0}\left(q_{0}\right), c_{0}\left(q_{2}\right), c_{2}(0)$, $c_{2}\left(q_{0}\right)$, and $c_{2}\left(q_{2}\right)$. Schematic, three-dimensional sections through this phase diagram are shown in Fig. 2 and were discussed earlier. Here we present detailed numerical results for two-dimensional sections through the phase diagrams of Fig. 2, on the basis of which Figs. 2(a) -2 (d) are drawn.

Consider first the section with $c_{0}\left(q_{2}\right)=c_{2}\left(q_{2}\right)=0$. In Fig. 4 we display phase diagrams in the $c_{0}\left(q_{0}\right)-c_{2}(0)$ plane for different values of $c_{2}\left(q_{0}\right)$. As we move up from negative values of $c_{2}\left(q_{0}\right)\left[c_{2}\left(q_{0}\right)=-0.01\right.$ in Fig. 4(a)] through $c_{2}\left(q_{0}\right)=0$ [Fig. $\left.4(\mathrm{~b})\right]$ to positive values of $c_{2}\left(q_{0}\right)$ $\left\{c_{2}\left(q_{0}\right)=0.1\right.$ in Fig. 4(c)], the phase bcc $O 1$ grows at the expense of $\operatorname{bcc} P, N$, and eventually $\boldsymbol{L}$ [cf. Figs. 2(a) $-2(\mathrm{~d})$ ]. For sufficiently large and negative $c_{2}\left(q_{0}\right)(<-0.01731$, the phase bcc $O 1$ lies outside the unit square in which we portray the phase diagram. Similarly, for sufficiently large and positive values of $c_{2}\left(q_{0}\right)$, the phases $\mathrm{N}$ and $\mathrm{bcc} P$ shrink and finally disappear [for $c_{2}\left(q_{0}\right)>0.212$ in the case of $\mathrm{N}$ and for $c_{2}\left(q_{0}\right)>0.130$ in the case of bccP]. Note that some of the phase boundaries in Fig. 4 are parallel to the $c_{0}\left(q_{0}\right)$ or $c_{2}(0)$ axes; this is an artifact of the approximations we have made.

We next consider the section with $c_{0}\left(q_{0}\right)=c_{0}\left(q_{2}\right)=0$ and plot the phase diagram in the $c_{2}\left(q_{2}\right)-c_{2}\left(q_{0}\right)$ plane for different values of $c_{2}(0): c_{2}(0)=0$ in Fig. 5(a) and $c_{2}(0)=0.5$ in Fig. 5(b). As $c_{2}(0)$ increases, the incommensurate bcc phase $\mathrm{IbccO}$ grows at the expense of bccO 1 , bccO2, and $\boldsymbol{L}$. [Recall that, on physical grounds, we expect $c_{2}\left(q_{2}\right)<c_{2}(0)$.]

Finally, consider the section with $c_{0}\left(q_{0}\right)=c_{0}\left(q_{2}\right)$ $=c_{2}\left(q_{0}\right)=0$. (This is the case most studied in various molecular-field theories of smectic ${ }^{10,36-39}$ and discotic ${ }^{40}$ ordering.) For these values of $c_{0}\left(q_{0}\right), c_{0}\left(q_{2}\right)$, and $c_{2}\left(q_{0}\right)$, we display in Fig. 6 the phase diagram in the $c_{2}\left(q_{2}\right)-c_{2}(0)$ plane. Solid lines indicate first-order phase boundaries.
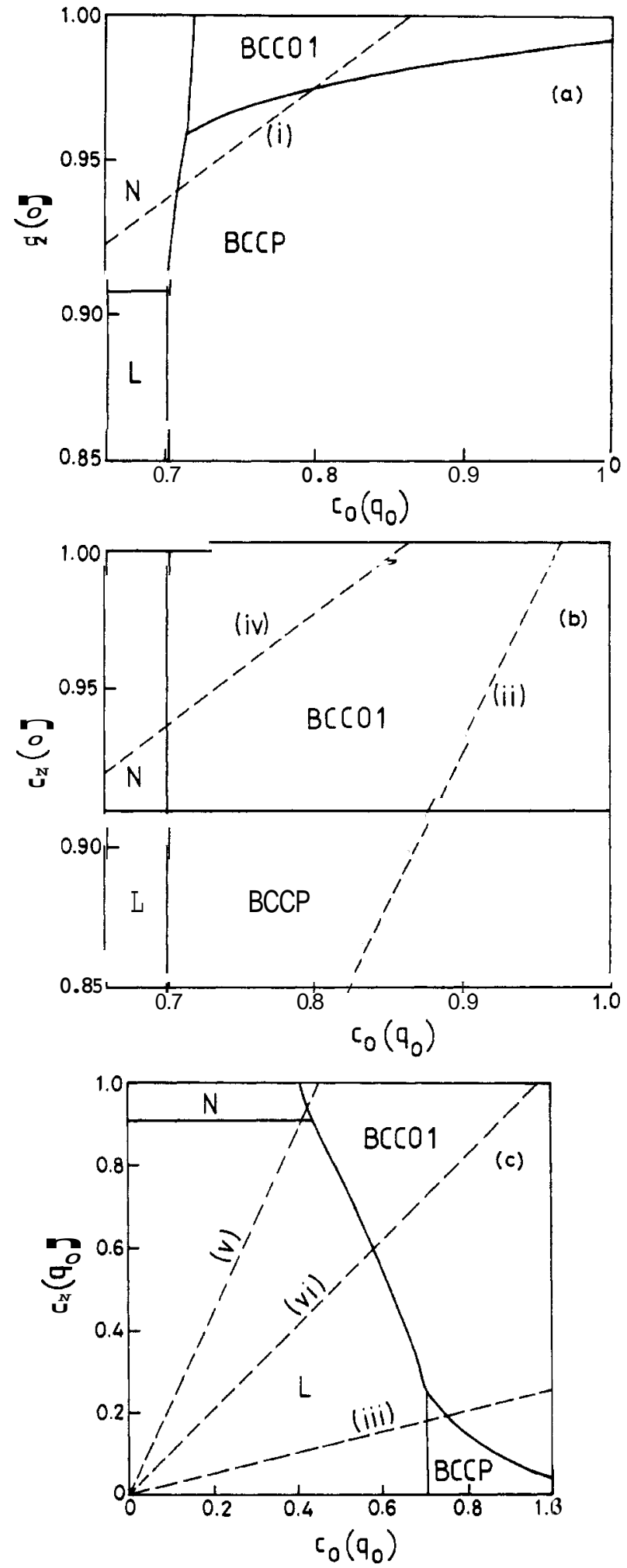

FIG. 4. Sections of the phase diagram in the $c_{0}\left(q_{0}\right)-c_{2}(0)$ plane for $c_{2}\left(q_{2}\right)=0, c_{0}\left(q_{2}\right)=0$, and varying $c_{2}\left(q_{0}\right)$. (a) $c_{2}\left(q_{0}\right)=-0.01,(\mathrm{~b}) c_{2}\left(q_{0}\right)=0$, and (c) $c_{2}\left(q_{0}\right)=0.1$. (See Table I for an explanation of the notations for the phases.) Note that in (a) and (b) the $L \cdot b c c P$ and the $\boldsymbol{L}-\boldsymbol{N}$ phase boundaries actually extend until $c_{2}(0)=0$ and $c_{0}\left(q_{0}\right)=0$, respectively. The dashed lines show in these figures are lines along which the order parameters (displayedin Fig. 8) have been calculated. 
Note that neither smectic nor discotic phases appear in this phase diagram; the transitions to smectic and discotic phases are always preempted by a transition to the orientationally ordered bcc phase bccO2. [For $c_{2}\left(q_{0}\right)>0$, bcc $O 1$ or $\mathrm{IbccO}$ might appear instead of bccO2 (Fig. 5), but never smectic or discotic phases.] The dashed (dot-dashed) line indicates where the liquidsmectic (liquid-discotic)phase boundary would have been had we not considered other ordered phases such as bccO2. The dashed line is precisely the phase boundary obtained by McMillan ${ }^{37}$ in his theory of smectic ordering. [In McMillan's theory, the nematic-smectic transition becomes continuous at a tricritical point; this tricritical point lies in the region $c_{2}(0)>1$, which we do not show in our phase diagrams as it violates the sum rule mentioned in Sec. I.]

We have already given (Sec. I) the basic reason for the metastability of smectic and discotic phases in our theory: once it becomes possible for the system to lower its free energy by forming oriented density waves with wave vector $q_{2}$, it goes into a phase which has the largest number of such density waves. Thus bccO2 (12 density waves) is thermodynamically stable whereas discotic ( 6 density waves) and smectic ( 2 density waves) are only metastable $\left(F_{\mathrm{Sm}}>F_{D}>F_{\mathrm{bccO} 2}\right)$; the bcc phase is further
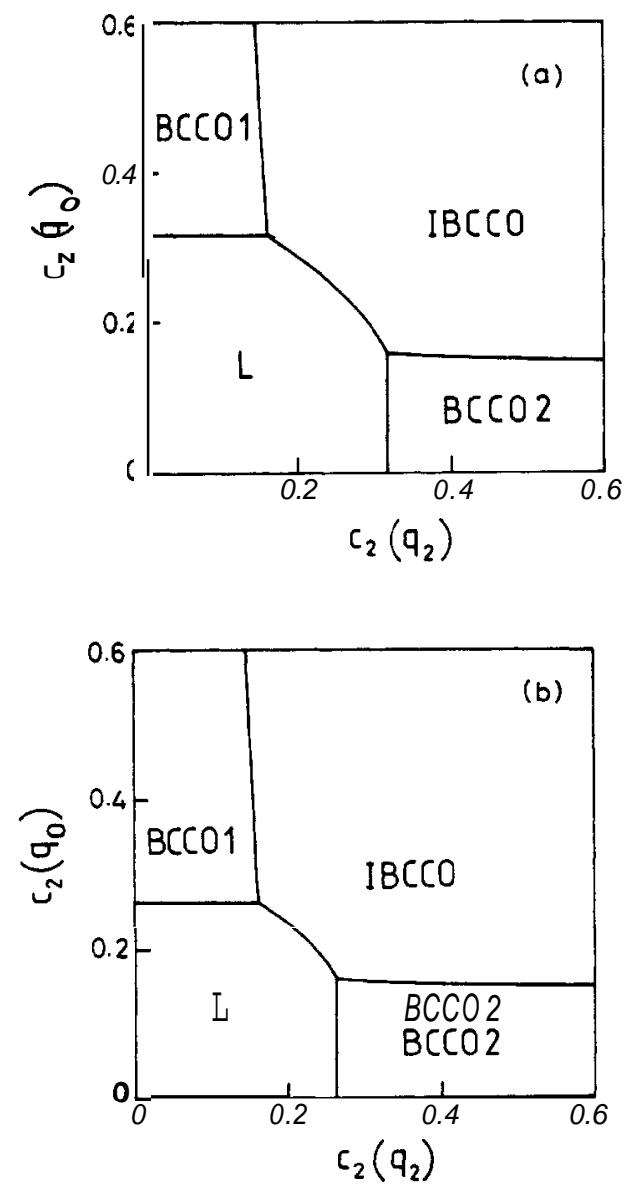

FIG. 5. Sections of the phase diagram in the $c_{2}\left(q_{2}\right)-c_{2}\left(q_{0}\right)$ plane for $c_{0}\left(q_{0}\right)=0, c_{0}\left(q_{2}\right)=0$, and varying $c_{2}(0)$. (a) $c_{2}(0)=0$ and $(b) c_{2}(0)=0.5$.

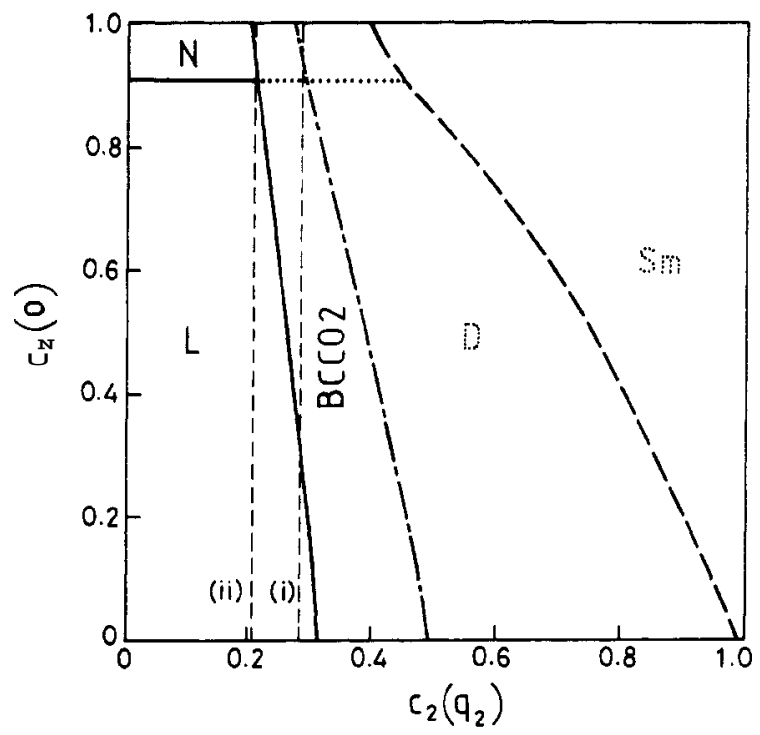

FIG. 6. Section of the phase diagram in the $c_{2}\left(q_{2}\right) \cdot c_{2}(0)$ plane for $c_{0}\left(q_{0}\right)=0, c_{0}\left(q_{2}\right)=0$, and $c_{2}\left(q_{0}\right)=0$. The solid line shows the actual phase boundaries for the $L$-bccO2, $\mathrm{N}$-bccO2, and the L-N phase transitions. The dot-dashed line and the dashed line show the "phase boundaries" at which the discotic $(D)$ and the smectic $(\mathrm{Sm})$ phases, respectively, become stable relative to the isotropic liquid $(L)$ and the nematic $(N)$. If the relations (14) are used, the L-Sm and $N . S m$ phase boundaries translate precisely to McMillan's (Ref. 37) phase boundaries. However, both the $\boldsymbol{D}$ and $\mathrm{Sm}$ phases are metastable relative to the bccO2 phase, hence the L-Sm and L-D phase boundaries are not real. The dotted line represents the $L-N$ phase boundary when both these phases are metastable with respect to bccO2. The thin dashed lines are lines along which the order parameters (displayed in Fig. 9) have been calculated.

stabilized by cubic terms in a Landau expansion of our free energy. By the same argument one might expect that the larger the number of such density waves in a phase the more it is disfavored by large and negative values of $c_{0}\left(q_{2}\right)$ (Fig. 1). Thus we have tried to find thermodynamically stable smectic and discotic phases in regions of the phase diagram where $c_{0}\left(q_{2}\right)$ is large and negative. However, we have met with no success: in Fig. 7 we plot the phase diagram in the $c_{0}\left(q_{2}\right)-c_{2}\left(q_{2}\right)$ plane, with $c_{0}\left(q_{0}\right)=c_{2}\left(q_{0}\right)=0$ and $c_{2}(0)=0.854$. (Solid, dashed, and dot-dashed lines have the same meanings as in Fig. 6.) As expected, the preempted L-D and L-Sm boundaries come close to the $L$-bcc 02 phase boundary as $c_{0}\left(q_{2}\right)$ becomes more and more negative; however, for sufficiently large and negative $c_{0}\left(q_{2}\right)$ (Fig. 7), these boundaries become parallel. Thus the $L$-bccO2 transition always preempts the L- $D$ and L-Sm transitions.

A word of caution: it is possible that smectic and discotic phases occupy some small volumes in the fivedimensional parameter space we investigate; and it is possible that we have missed these volumes. However, these volumes are certainly not as large as indicated by previous molecular-field theory calculations. ${ }^{10,36-40}$

All the phase boundaries in our phase diagrams are 


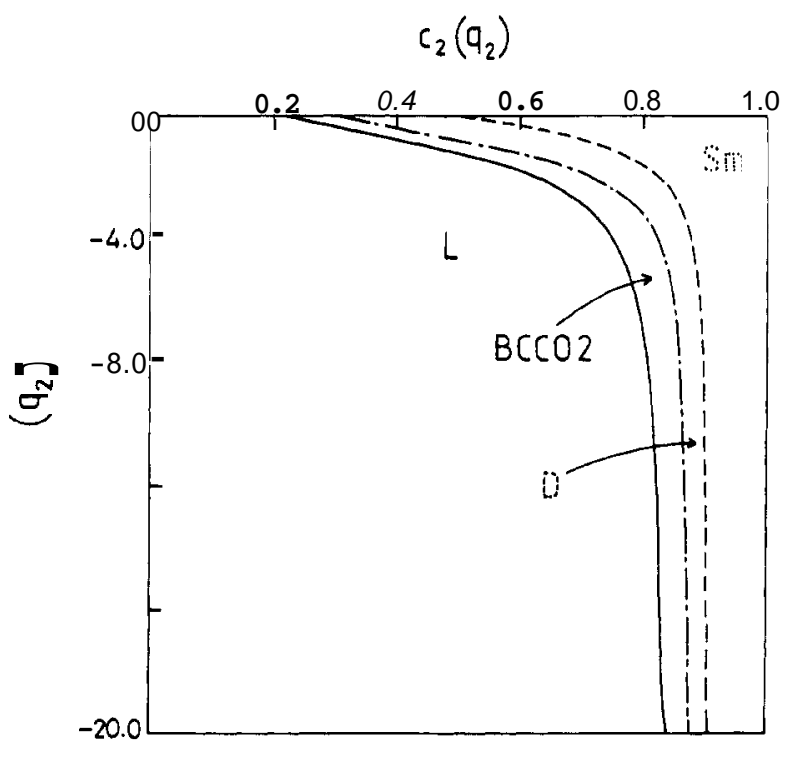

FIG. 7. Section of the phase diagram in the $c_{0}\left(q_{2}\right)-c_{2}\left(q_{2}\right)$ plane with $c_{0}\left(q_{0}\right)=0, c_{2}\left(q_{0}\right)=0$, and $c_{2}(0)=0.854$, showing the effect of large negative $c_{0}\left(q_{2}\right)$ on the $L$-bccO2 phase boundary (__ ) and the $L-D(-\cdot-\cdot-\cdot)$, and L-Sm $\left({ }_{-}-\right)$, "phase boundaries" (Fig.6).

first-order boundaries. Order parameters jump at such boundaries, as does the entropy. To calculate the jump in the entropy we need to know the dependence of the direct correlation function on temperature. ${ }^{51}$ This is not available for any mesogenic system, so we do not calculate the entropy jumps at various transitions. However, the jumps in the order parameters follow simply from our calculations. In Figs. 8 and 9 we show representative plots of the variation of the order parameters $\tau_{2}(0)$, etc., along chosen lines in the parameter space.

In Fig. 8(a) we show how the order parameters $\tau_{2}(0)$, $\tau_{2}\left(q_{0}\right)$, and $\tau_{0}\left(q_{0}\right)$ vary along the line $c_{2}(0)=0.389 c_{0}\left(q_{0}\right)$ +0.664 shown in the phase diagram of Fig. 4(a). Note that the sequence of transitions $\mathrm{L} \rightarrow \mathrm{N} \rightarrow$ bcc $P \rightarrow$ bccO 1 is obtained as in some recent experiments. ${ }^{43}$ (In these experiments, the structure of the orientationally ordered crystal is not determined.) The sequence of transitions $L$-bccP-bcc $O 1$ and $L-N$-bccO 1 are obtained easily by moving along straight lines in the phase diagrams of Fig. 4. Figures $8(\mathrm{~b})\left[c_{2}\left(q_{0}\right)=0\right]$ and $8(\mathrm{c})\left[c_{2}\left(q_{0}\right)=0.1\right]$ show representative plots of the order parameters along such lines for the sequence $L$-bccP-bccO 1 ; Figs. $8(\mathrm{~d})$ and $8(\mathrm{e})$ show analogous plots for the sequence $L-N$-bcc $O 1$. Note that the order parameter $\tau_{0}\left(q_{0}\right)\left[\tau_{2}(0)\right]$ does not jump at the bcc $P$-bccO $1(N$-bcc $O 1)$ transition if $c_{2}\left(q_{0}\right)=0$; this is a nongeneric feature which is not present if $c_{2}\left(q_{0}\right) \neq 0$. In Fig. 8(f) we show an example of a direct $L$-bcc $O 1$ transition. Figures $9(\mathrm{a})$ and $9(\mathrm{~b})$ show two scans through the phase diagram of Fig. 6 . In the first $\left[c_{2}\left(q_{2}\right)=0.284\right]$, a direct $L$-bccO2 transition is obtained; in the second $\left[c_{2}\left(q_{2}\right)=0.209\right]$, the sequence $L \cdot N$-bcc $O 2$ is obtained. In $\mathrm{Sec}$. IV we compare the results summarized in this sec- tion with numerical simulations of the experiments on mesogenic systems.

\section{COMPARISON WITH NUMERICAL SIMULATIONS AND EXPERIMENTS}

To compare the results of Sec. III with those of numerical simulations and experiments, we need to know how the direct correlation function changes as the pressure, temperature, etc., are changed. However, this is not known reliably for any mesogenic systems either from experiments of from numerical simulations (e,g, simulations of a system of hard ellipsoids of revolution).

We attempt here to compare our results with those of computer simulations of a system of hard ellipsoids of revolution by following the method of Singh and Singh: ${ }^{2}$ we use a simple, but crude, approximation for the direct correlation function of a system of hard ellipsoids of revolution. We approximate this system by a hard-sphere system with an effective, hard-sphere diameter $\boldsymbol{D}$ given by the Gaussian-overlap model of Berne and Pechukas, ${ }^{52}$

$$
\begin{array}{r}
D\left(\hat{\mathbf{r}}_{12}, \hat{\mathbf{\Omega}}_{1}, \hat{\mathbf{\Omega}}_{2}\right)=D_{0}\left(1-\frac{x}{1-x^{2}\left(\hat{\mathbf{\Omega}}_{1} \cdot \hat{\mathbf{\Omega}}_{2}\right)^{2}}\right. \\
\quad \times\left[\left(\hat{\mathbf{r}}_{12} \cdot \hat{\mathbf{\Omega}}_{1}\right)^{2}+\left(\hat{\mathbf{r}}_{12} \cdot \hat{\mathbf{\Omega}}_{2}\right)^{2}\right. \\
\quad-2 x\left(\hat{\mathbf{r}}_{12} \cdot \hat{\mathbf{\Omega}}_{1}\right)\left(\hat{\mathbf{r}}_{12} \cdot \hat{\mathbf{\Omega}}_{2}\right) \\
\left.\left.\quad \times\left(\hat{\mathbf{\Omega}}_{1} \cdot \hat{\mathbf{\Omega}}_{2}\right)\right]\right)^{-1 / 2},
\end{array}
$$

where

$$
\begin{aligned}
& \mathrm{x}=\left(a^{2}-b^{2}\right) /\left(a^{2}+b^{2}\right), \\
& D_{0}=2 b,
\end{aligned}
$$

and $a$ and $b$ are, respectively, the lengths of the semimajor and semiminor axes of the ellipsoids of revolution. We next approximate the direct correlation function by the form it assumes for hard spheres in the PercusYevick approximation, ${ }^{53}$ namely,

$$
c\left(\mathbf{r}_{12}, \hat{\mathbf{\Omega}}_{1}, \hat{\mathbf{\Omega}}_{2}\right)=\left\{\begin{array}{l}
A+B\left(r_{12} / D\right)+A(\eta / 2)\left(r_{12} / D\right)^{3}, \\
r_{12} / D<1, \\
0, \quad r_{12} / D>1,
\end{array}\right.
$$

where

$$
\begin{aligned}
& A=-(1+2 \eta)^{2} /(1-\eta)^{4}, \\
& B=6[1+(\eta / 2)]^{2} /(1-\eta)^{4}
\end{aligned}
$$

$\eta$ is the packing fraction. In Eq. (16a) all of the dependence of $c$ on $\widehat{\mathbf{r}}_{12}, \widehat{\boldsymbol{\Omega}}_{1}$, and $\widehat{\boldsymbol{\Omega}}_{2}$ comes from $\boldsymbol{D}$ [Eq. (15a)]. This approximation for $c\left(\mathbf{r}_{12}, \widehat{\mathbf{\Omega}}_{1}, \hat{\Omega}_{2}\right)$ should be reasonable at low values of the packing fraction $\eta$ and for $a / b \simeq 1$. (The more eccentric the ellipsoids, the lower must $\eta$ be for the validity of this approximation.)

We use the approximation (16) to calculate $c_{0}\left(q_{0}\right)$, 

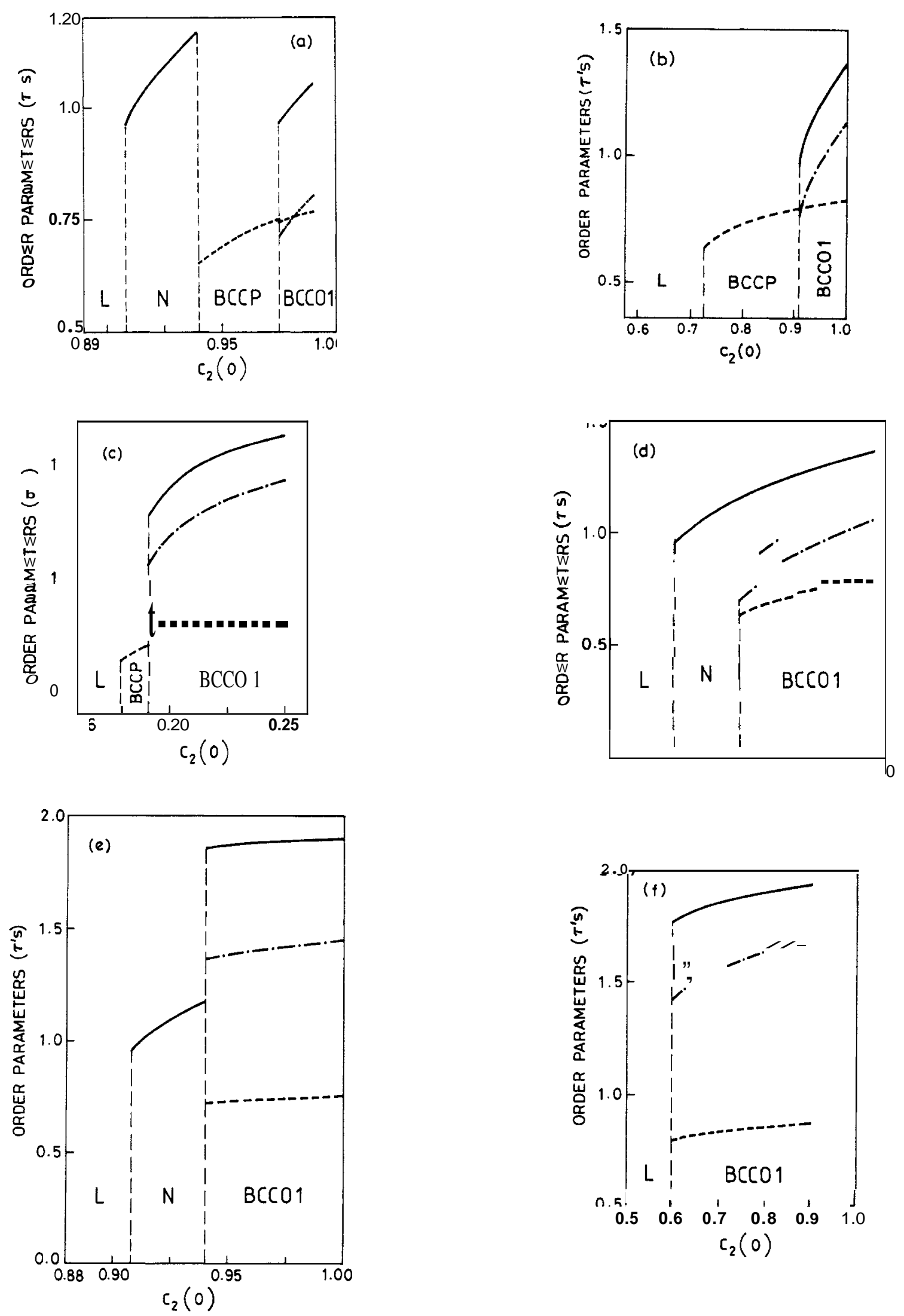

FIG. 8. Variation of the order parameters $\tau_{2}(0)$ (solid line), $\tau_{0}\left(q_{0}\right)$ (dashed line), and $\tau_{2}\left(q_{0}\right)$ (dot-dashed line) along the various lines marked in the phase diagrams of Fig. 4. (a)line (i) in Fig. 4(a); (b) line (ii) in Fig. 4(b); (c) line (iii) in Fig. 4(c); (d) line (iv) in Fig. 4(b); (e) line (v) in Fig. 4(c); (f) line (vi) in Fig. 4(c). 
$c_{2}(0)$, etc., as functions of $\eta$ and $a / b$. We then convert the phase diagrams of Sec. III into an $\eta-a / b$ phase diagram such as the one obtained from numerical simulati o n ' ' (Fig. 3). Figure 10 shows the $\eta-a / b$ phase diagram we obtain. We restrict ourselves to the region $a / b>1$ because, at the level of our approximation (16), this phase diagram is symmetric about the line $a / b=1$ under the replacement $a / b \rightarrow b / a$. Shaded regions in Fig. 10 indicate where the approximation (16) leads to unphysical values of $c_{2}(0)$, etc., such as $c_{2}(0)>1$. Note that only the phases $L, b c c P$, and $N$ appear in the phase diagram of Fig. 10. This is because one (or more) of the
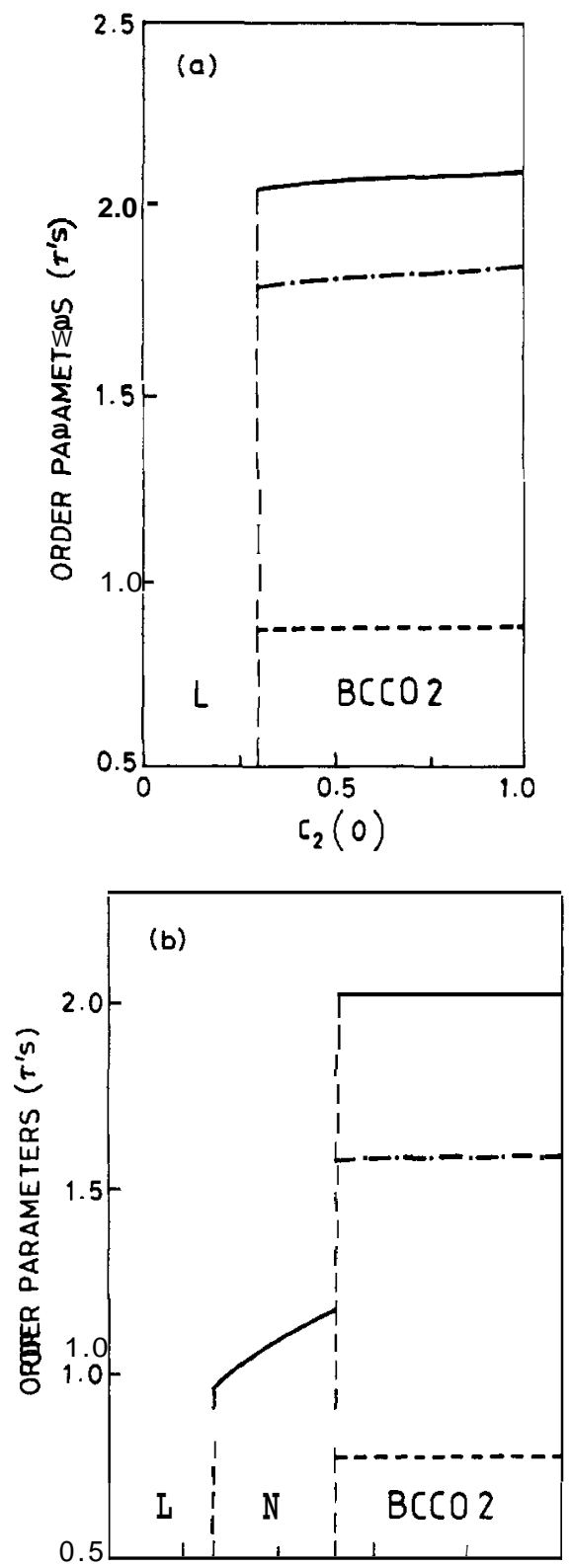

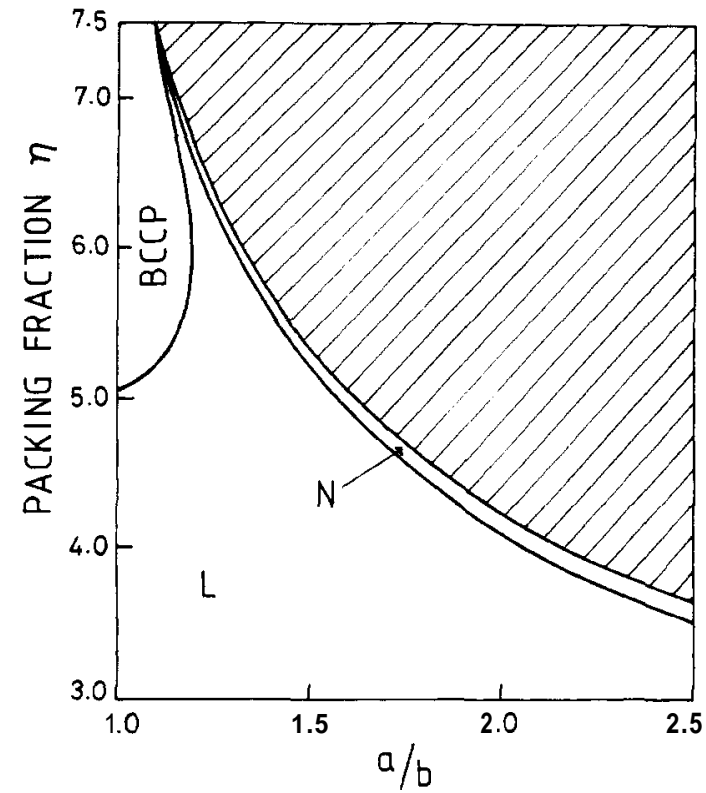

FIG. 10. Calculated phase diagram in the $\eta$ (packing fraction)-a/b plane for a system of hard ellipsoids of revolution. This phase diagram is obtained from the phase diagrams of Figs. 4-7 using the approximations discussed in Sec. IV [Eqs. (15) and (16)] for the direct correlation function.

$c_{2}$ 's and $c_{0}$ 's assume an unphysical value [because of approximation (16)] before the other phases shown in Fig. 2 are stabilized.

There is fair qualitative agreement between the $L$-bcc $P$ and $\boldsymbol{L} \cdot \boldsymbol{N}$ phase boundaries in the phase diagrams of Fig. 3 (computer simulations) and Fig. 10 (our calculation). (Note, however, that the crystalline phases obtained in the numerical simulations which yield Fig. 3 have fcc or distorted fcc structures, whereas we only consider bcc ordering.) Another point of agreement between our study and the computer simulations ${ }^{1,29}$ is that neither yields thermodynamically stable smectic or discotic phases.

Clearly we need a better approximation than Eq. (16) for $\boldsymbol{c}\left(\mathbf{r}_{12}, \widehat{\mathbf{\Omega}}_{1}, \hat{\mathbf{\Omega}}_{2}\right)$ to uncover what lies in the shaded region of Fig. 10 and then to compare it with Fig. 3. However, as pointed out earlier, given the approximate calculations we describe in Secs. II and III, no matter what approximation we use for $c\left(\mathbf{r}_{12}, \widehat{\mathbf{\Omega}}_{1}, \hat{\mathbf{\Omega}}_{2}\right)$ we cannot obtain smectic or discotic phases (Sec. III) except as metastable states. Also, the orientational order parameter jumps discontinuously at the bccP-orientationally-ordered-solid transition in our theory, whereas it changes continuously in a system of hard ellipsoids of revolution. ${ }^{54}$

Very recent simulations ${ }^{30}$ on a system of parallel hard spherocylinders have yielded a smectic phase. We have not tried to compare our phase diagrams (Secs. I and III) with those obtained from these simulations. To do so we would need an approximation such as (16) for a system of parallel hard spherocylinders. Our calculations apart, it is puzzling that very similar simulations of systems of hard ellipsoids of revolution ${ }^{29}$ and parallel hard spherocylinders $^{30}$ yield qualitatively different results, namely, the former shows no stable smectic phase whereas the latter does. 
As we have mentioned previously (Secs. I and III) it is not easy to compare our phase diagrams with those determined experimentally. However, even a crude comparison is instructive, though often embarrassing, for our theory as well as for all molecular-field theories, ${ }^{3,36-40}$ that are special cases of it. We have mentioned many times the absence of stable smectic and discotic phases in our theory. We list some other points in the following.

Though the crystalline phases of mesogenic systems have not been studied as thoroughly as their liquidcrystalline phases, it is well known ${ }^{14,15}$ that the crystalline phases often have structures that have a much lower symmetry than the bcc phases we consider. Given the approximations we have made, it is not possible for us to obtain such low-symmetry crystalline phases as thermodynamically stable phases. However, it would be interesting to look for incommensurate crystalline phases (like $I b c c O$, but perhaps with lower symmetry) in mesogenic systems. (Incommensurate smectics have been observed recently. ${ }^{55}$ ) We should warn the reader, though, that the approximations we have made (Secs. II and III) overestimate the stability of incommensurate phases. ${ }^{56}$

Another experimental observation that deserves explanation is the phase diagram shown in Fig. 11. It shows how the transition temperatures for various transitions in mesogenic systems behave as a function of the length of the alkyl-chain tail of the mesogenic molecules that comprise the system. In most cases $^{57} T_{L \cdot N}$, the tran-
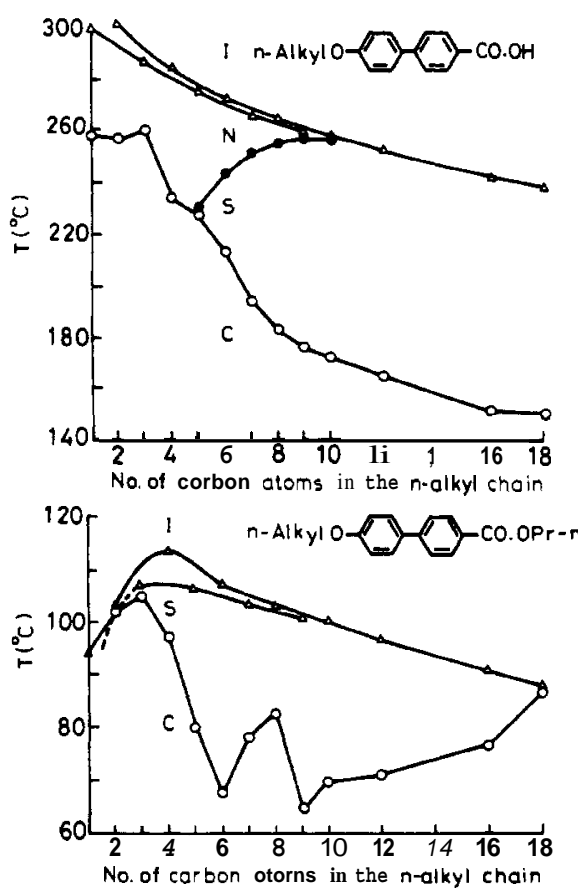

FIG. 11. Experimental plots of transition temperatures vs chain length for two homologous series of mesogenic compounds. The symbols $\mathrm{I}, N, \mathrm{~S}$, and $C$ represent, respectively, the isotropic liquid, nematic, smectic and crystalline phases (Ref. 57). Transition temperatures for $I-N$ and $I-S$ transitions are indicated by triangles, for $N-S$ transitions by closed circles, and for $N \cdot C$ and $S \cdot C$ transitions by open circles. Lines are drawn through data points to guide the eye. sition temperature for the liquid-nematic transition, $\boldsymbol{d e}$ creases as $\boldsymbol{n}$, the length of the alkyl-chain tail, increases! This is contrary to what we would expect on the basis of our theory (Fig. 10) and computer simulations of systems of hard ellipsoids of revolution (Fig. 3): in both of these studies $T_{L-N}$ increases as $a / b$, the eccentricity of the ellipsoids, increases (for decreasing, the packing fraction is equivalent to increasing the temperature ${ }^{33}$ ). The reason for this discrepancy is obvious: we cannot hope to model long alkyl-chain tails which areflexible by increasing the eccentricity of ellipsoids that are hard and completely inflexible. Some molecular-level theories have been proposed $^{58}$ to explain phase diagrams such as the one shown in Fig. 11; however, these theories do not consider positionally ordered phases. Unfortunately, it is not clear how to put in information about the flexibility of alkylchain tails $\mathbf{s}^{57}$ into the direction correlation function we use.

In experimental mesogenic systems the transition from a plastic crystal to an orientationally ordered crystal is of first order. ${ }^{59}$ At this transition the jump in the entropy is often substantially lower than the jump in the entropy at the liquid-plastic crystal transition. ${ }^{59}$ Density-functional theories, such as ours and that of McMullen and Oxtoby, ${ }^{13}$ can be used, in principle, to calculate these entropy jumps. ${ }^{51}$ However, such calculations can hardly be expected to give numerically reliable results, for neither our study nor that of McMullen and Oxtoby ${ }^{13}$ allows for a change in the crystal structure at the transition from the plastic crystal to the orientationally ordered crystal; such a change in the crystal structure occurs almost invariably at this transition. ${ }^{59}$

\section{CONCLUDING REMARKS}

We have listed in the preceding various discrepancies between our theory (and other molecular-field theories that are special cases of it) and experiments on and simulations of mesogenic systems. These discrepancies presumably arise because of the various approximations we make.

(1) We neglect high-order correlation functions $\left[c^{(n)}=0\right.$ for $\left.n \geq 3\right]$.

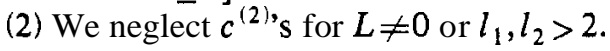

(3) We parametrize the functions $c_{0}(G)$ and $c_{2}(G)$ [Eq. (2)] in terms of their values at $G=0, q_{0}$, and $q_{2}$; at all other values of $G$ these functions are taken to be zero, and the fluid is assumed incompressible, i.e., $c_{0}(0)=-\infty$.

(4) We allow for only one form of three-dimensional ordering, namely bcc.

(5) We restrict ourselves to mesogenic systems in which the molecules have a center-of-inversion symmetry (this is usually not the case).

(6) We neglect effects of fluctuations on the order parameters we use.

It is not easy to tell precisely which of the shortcomings of our theory follow from which one of the approximations we have made. Consequently, it is difficult to decide how best to improve on our theory (and all other molecular-field theories which are special cases of it), especially because the removal of most of the approximations listed in the preceding involves substantial efforts. 
These limitations notwithstanding, we believe that the theoretical framework presented in this paper is superior to earlier molecular-field theories for phase transitions in mesogenic systems and removes some of their obviously unsatisfactory features.

For example, consider the isotropic-nematic transition. According to the theory presented here this transition takes place when $c_{2}(0)=0.908$. The transition temperature, the entropy jump at the transition, and the temperature dependence of properties below the transition are determined by the temperature dependence of $c_{2}(0)$. In molecular-field theories, e.g., McMillan's, ${ }^{37} c_{2}(0)$ is simply parametrized as $V_{0} /\left(5 k_{B} T\right)$, where $V_{0}$ is a molecular energy scale. Hence the transition temperature is $T_{N-L}=0.2203 V_{0} / k_{B}$; the order parameter $\tau_{2}^{*}(0)$ at the transition, equal to 0.95936 , and the entropy jump at the transition," given by

$$
-k_{B}\left[\tau_{2}^{*}(0)\right]^{2}\left(V_{0} / 10 k_{B} T_{N-L}\right)=-1.0133 k_{B},
$$

is universal; and properties below the transition, which depend on $V_{0} /\left(k_{B} T\right)$ a $T / T_{N-L}$, are universal functions of $T / T_{N-L}$. None of these results of conventional molecular-field theories is in agreement with experiments. Our theory does not lead to such disagreement. Of course, fluctuations must be included for an accurate description of isotropic-nematic transitions since these are weakly first order. This is beyond the scope of this study.

Conventional molecular-field theories ${ }^{10,36-40}$ do not allow for volume or density changes at mesophase transitions. We can allow for such changes quite simply by relaxing our incompressibility assumption, i.e, by allowing $c_{0}(0)$ to be finite.

To understand the systematics of the dependence of the transition temperatures, the entropy jumps at the transitions, and properties in the ordered phases on molecular lengths (or diameters), we must know how the Fourier coefficients of the direct correlation function, such as $c_{0}(q)$ and $c_{2}(q)$, depend on molecular lengths (especially the lengths of the flexible alkyl-chain parts) or diameters. To the best of our knowledge, it is not known how $c_{0}(q)$, etc., depend on these lengths. Thus some of the detailed comparisons that have been made between earlier molecular-field theories and experimental results are of questionable significance. $^{60}$

Finally, our study brings into sharp focus a big flaw of conventional molecular-field theories: ${ }^{10,36-40}$ They cannot account for the thermodynamic stability of smectic or discotic phases (relative to three-dimensional orienta- tionally ordered crystalline phases). To explain the stability of smectic and discotic phases is one of the major challenges that mean-field theories of mesophases must face.

It is likely that two features not included in our theory may be responsible for stabilizing smectics and discotics. (1) The detailed $q$ dependence of $c_{l}(q)$ may be important. [So we might have to allow for nonzero $c_{l}(G)$ at many more values of $G$ than we have done here.] (2) It may be necessary to include Fourier components $c_{l l^{\prime} L}(q)$, with $\mathrm{L} \neq 0$, which couple the directions of orientational ordering and positional ordering. We hope to present elsewhere the results of studies investigating these possibilities.

In conclusion, we want to point out that detailed experimental studies of phases (including crystalline phases) and phase transitions in mesogenic systems subject to very strong electric and magnetic fields should shed a great deal of light on many of the questions raised in this paper. For, with such fields, mesogenic molecules become orientationally aligned and, for sufficiently strong fields, we can assume that they are fully aligned in the nematic phase. Then, within the framework of our theory, the transitions to positionally ordered (smectic, discotic, or crystalline) phases are determined by the direct correlation function $c^{(2)}\left(\mathbf{r}_{12} ; \widehat{\mathbf{z}}, \widehat{\mathbf{z}}\right)$ (assuming the $z$ axis to be along the alignment direction), which can be thought of as an anisotropic, but purely positiondependent, direct correlation function. Much more can be said about such an anisotropic correlation function in a fully oriented system than about $c^{(2)}\left(\mathbf{r}_{12} ; \widehat{\mathbf{\Omega}}_{1}, \widehat{\mathbf{\Omega}}_{2}\right)$. For example, Lebowitz and Perram ${ }^{61}$ have shown how to calculate such functions for a system of oriented, hard ellipsoids of revolution ${ }^{62}$ using the Percus-Yevick approximation. Also, such a function can be measured by scattering neutrons off strongly oriented mesogenic systems. Thus one can study experimentally and theoretically the systematics of the relationship between this anisotropic direct correlation function and the phase transitions between various positionally ordered phases.

\section{ACKNOWLEDGMENTS}

We would like to thank S. V. Bhat, N. Madhusudana, T. V. Ramakrishnan, R. Shashidhar, and Y. Singh for various useful discussions. We would also like to thank the University Grants Commission (India), the Department of Science and Technology (India), and the Council for Scientific and Industrial Research (India)for support.
${ }^{1} \mathrm{P}$. G. deGennes, The Physics of Liquid Crystals (Clarendon, Oxford, 1974).

${ }^{2}$ S. Chandrasekhar, Liquid Crystals (Cambridge University Press, Cambridge, England, 1977).

${ }^{3}$ The Molecular Physics of Liquid Crystals, edited by G. R. Luckhurst and G.W. Gray (Academic, New York, 1974).

${ }^{4}$ T. V. Ramakrishnan and M. Yussouff, Phys. Rev. B 19, 2775 (1979).

${ }^{5}$ T. V. Ramakrishnan, Pramana 22,365 (1984).
${ }^{6}$ A. D. J. Haymet and D. W. Oxtoby, J. Chem. Phys. 74, 2559 (1981).

${ }^{7}$ P. Tarazona, Mol. Phys. 52, 81 (1984).

${ }^{8}$ G. L. Jones and U. Mohanty, Mol. Phys. 54, 1241 (1985).

${ }^{9}$ For a recent review and bibliography see A. D. J. Haymet, in Ann. Rev. Phys. Chem. (to be published). Density-functional theories have also been used to study quasicrystalline ordering [S. Sachdev and D. R. Nelson, Phys. Rev. B 32, 4592 (1985)] and frozen disordered solids [Y. Singh, J. P. Stoessel, 
and P. G. Wolynes, Phys. Rev. Lett. 54, 1059(1985)].

${ }^{10}$ F. T. Lee, H. T. Tan, and C. W. Woo, Phys. Lett. 48A, 68 (1974).

${ }^{11} \mathrm{D}$. Frenkel and B. Mulder, Mol. Phys. 55, 1171(1985).

${ }^{12}$ U. P. Singh and Y. Singh, Phys. Rev. A 33, 2725 (1986).

${ }^{13}$ W. F. McMullen and D. W. Oxtoby, J. Chem. Phys. 86, 4146 (1987).

${ }^{14}$ Liquid Crystals and Plastic Crystals, edited by G. W. Gray and P. A. Winsor (Ellis Horwood, Chichester, England, 1974), Vol. 1.

${ }^{15} \mathrm{G}$. W. Gray and J. W. Goodby, Smectic Liquid Crystals (Leonard Hill, London, 1984),

${ }^{16}$ S. Chandrasekhar, B. K. Sadashiva, and K. A. Suresh, Pramana 9,471 (1977).

${ }^{17}$ We consider only those discotics that have hexagonal twodimensional order. Some laboratory discotics actually order in square lattices.

${ }^{18}$ The Plastic Crystalline State, edited by J. N. Sherwood (Wiley, New York, 1979).

${ }^{19}$ In the plastic crystalline phase we consider the tips of the mesogenic molecules to lie anywhere on the surface of a sphere; however, in laboratory plastic crystals there is a finite number of equivalent orientations between which the molecules flip.

${ }^{20} \mathrm{C}$. Zannoni, in The Molecular Physics of Liquid Crystals, Ref. 3, pp. 191-220.

21 J. Viellard-Baron, J. Chem. Phys. 56, 4729 (1972).

22J. Viellard-Baron, Mol. Phys. 28, 809 (1974).

${ }^{23}$ H. J. F. Jansen, G. Vertogen, and J. G. J. Ypma, Mol. Cryst. Liq. Cryst. 38, 87 (1977).

${ }^{24}$ P. A. Lebwohl and G. Lasher, Phys. Rev. A 6,426(1972).

${ }^{25}$ G. R. Luckhurst and S. Romano, Mol. Phys. 40, 129 (1980).

${ }^{26}$ J. Y. Denham, G. R. Luckhurst, C. Zannoni, and J. W. Lewis, Mol. Cryst. Liq. Cryst. 60, 185 (1980).

${ }^{27}$ G. R. Luckhurst and S. Romano, Proc. R. Soc. London, Ser. A 373, 111 (1980).

${ }^{28}$ G. R. Luckhurst, P. Simpson, and C. Zannoni, Chem. Phys. Lett. 78,429 (1981).

${ }^{29}$ D. Frenkel, B. Mulder, and J. P. McTague, Phys. Rev. Lett. 52, 287 (1984);B. Mulder and D. Frenkel, Mol. Phys. 55, 1193 (1985);D. Frenkel, ibid. 60, 1 (1987).

${ }^{30}$ A. Stroobants, H. N. W. Lekkerkerker, and D. Frenkel, Phys. Rev. Lett. 57, 1452(1986).

${ }^{31}$ M. P. Allen and A. A. Imbriesrki, Mol. Phys. 60,453 (1987).

${ }^{32}$ D. L. Johnson, J. Chem. Phys. 8, 45 (1983).

${ }^{33} \mathrm{~J}$. P. Hansen and I. R. MacDonald, Theory of Simple Liquids (Academic, New York, 1976).

${ }^{34} \mathrm{As}$ we will show later, $c_{2}\left(q_{2}\right)$ promotes smectic ordering with wave vector $q_{2}$. Hence $q_{2}=2 \pi / d$, where $d$ is the length of the molecule. $c_{0}\left(q_{0}\right)$ promotes bcc plastic crystalline ordering with $q_{0}$ as the smallest reciprocal vector. If we assume that in the bcc plastic crystal the spherical surfaces on which tips of the nearest-neighbor molecules move almost touch, then $q_{0}=(2 \pi / d)\left(\frac{3}{2}\right)^{1 / 2}=(1.5)^{1 / 2} q_{2}$.

${ }^{35}$ The phase diagrams of Fig. 2 show lines of four-phase coexistence and points of five- and six-phase coexistence. Normally such lines and points do not occur in phase diagrams in a three-dimensional parameter space. These nongeneric features occur in the phase diagrams of Fig. 2 at very special sections [Eq. (1) and the restrictions $c_{0}(G)=c_{2}(G)=0$ for $\mathrm{G}=O, q_{0}$, and $q_{2}$ ] through an infinite-dimensional parameter space [of the functions $c_{l, l, L}(q)$ ]. We expect such nongeneric features to be absent in a generic section [at the simplest lev- el, a section in which $c_{l_{1} l_{2} L}(0), c_{l_{1} l_{2} L}\left(q_{0}\right)$, and $c_{l_{1} l_{2} L}\left(q_{2}\right)$ are small in magnitude, but fixed at some nonzero value, for $l_{1}, l_{2}, L>2$ ]. Indeed, even as we move from the nongeneric section of Figs. 2(b) $\left[c_{2}\left(q_{0}\right)=0\right]$ to the less nongeneric sections of Figs. 2(a) and 2(c) $\left[c_{2}\left(q_{0}\right) \neq 0\right]$, we see that one line of four-phase coexistence (between the phases $L, N$, bcc $P$, and bcc $O 1$ ) is replaced by a first-order phase boundary ending in lines of three-phase coexistence.

${ }^{36}$ K. K. Kobayashi, Phys. Lett. 31A, 125 (1970); J. Phys. Soc. Jpn. 29, 101 (1970);Mol. Cryst. Liq. Cryst. 13, 137 (1971).

${ }^{37}$ W. L. McMillan, Phys. Rev. A 4, 1238 (1971);6, 936 (1972);7, 1419(1973);7, 1673(1973); 8, 328 (1973).

${ }^{38}$ F. T. Lee, H. T. Tan, Y. M. Shih, and C. W. Woo, Phys. Rev. Lett. 31, 1117 (1973).

${ }^{39}$ G. F. Kventsel, G. R. Luckhurst, and H. B. Zwedie, Mol. Phys. 56, 589 (1985).

40S. Chandrasekhar, Philos. Trans. R. Soc. London, Ser. A 309, 93 (1983).

${ }^{41}$ P. Harrowell and D. W. Oxtoby, Mol. Phys. 54, 1325(1985).

${ }^{42}$ See, e.g., the review by S. Chandrasekhar and R. Shashidhar in Advances in Liquid Crystals, edited by G. H. Brown (Academic, New York, 1970), Vol. 4, p. 83.

${ }^{43}$ S. Arumugam, S. V. Bhat, N. Kumar, K. V. Ramanathan, and R. Srinivasan, Mol. Cryst. Liq. Cryst. 126, 161 (1985).

${ }^{44}$ M. Raj Lakshmi, H. R. Krishnamurthy, and T. V. Ramakrishnan (unpublished).

${ }^{45}$ T. J. Sluckin and P. Shukla, J. Phys. A 16, 1539(1983).

${ }^{46}$ M. D. Lipkin and D. W. Oxtoby, J. Chem. Phys. 79, 1939 (1983).

${ }^{47}$ For a comparison of these approximations in a slightly different context, see C. Dasgupta and R. Pandit, J. Stat. Phys. 47, 375 (1987).

${ }^{48}$ W. Maier and A. Saupe, Z. Naturforsch. 13A, 564 (1958);14A, 882 (1958)

${ }^{49} \mathrm{C}$. Zannoni, in The Molecular Physics of Liquid Crystals, Ref. 3, pp. 51-83.

${ }^{50}$ M. E. Rose, Elementary Theory of Angular Momentum (Wiley, New York, 1957).

${ }^{51}$ From (11) it is not hard to show that the entropy in the ordered phase is given by

$$
\begin{aligned}
S-S_{\mathrm{liq}}= & -\left(F-F_{\mathrm{liq}}\right) / T \\
& +\left(\rho_{\mathrm{liq}} V k_{B} T / 2\right) \sum_{\mathrm{G}} \sum_{l}\left[\tau_{l}(G)\right]^{2} \frac{d c_{l}}{d T}(G) .
\end{aligned}
$$

Right at the transition from the liquid to the ordered phase the first term is zero, and the entropy jump is given by the second term alone. Typically $d c_{l}(G) / d T<0\left[c_{l}(G)\right.$ increases as Tdecreases], hence $S<S_{\text {liq }}$, as it should be.

52 B. J. Berne and P. Pechukas, J. Chem. Phys. 56,4213 (1972).

$53 \mathrm{~J}$. P. Hausen and I. R. MacDonald, Theory of Simple Liquids, Ref. 33, pp. 116-123.

${ }^{54}$ In Refs. 11 and 29 this continuous change in the orientational order parameter and a similar change in the density is given as evidence for the continuous nature of the plastic. crystal-to-orientationally-ordered-solid transition. We believe that this transition must really be a first-order transition because the order parameter characterizing the new positional order must change discontinuously. (The crystal structures of the plastic-crystal and orientationally-ordered-crystal phases are different.)

${ }^{55}$ B. R. Ratna, R. Shashidhar, and V. N. Raja, Phys. Rev. Lett. 55, 1476(1985). 
${ }^{56}$ In a more complete theory, density waves of very small wave vectors (arising from large-order combinations of the basic incommensurate wave vectors) have to be considered. These cost energy because of the large negative $c_{0}(q)$ for small $q$, and hence reduce the stability of incommensurate phases. See Sachdev and Nelson (Ref. 9) for a discussion of this point in the context of quasicrystals.

${ }^{57}$ G. W. Gray, in Liquid Crystals and Plastic Crystals, Ref. 14,p. 117; G. W. Gray, J. B. Hartley, and B. Jones, J. Chem. Soc. Pt. II, 1412 (1955).

${ }^{58}$ S. Marcelja, J. Chem. Phys. 60, 3599 (1974).

${ }^{59}$ P. A. Winsor, in Liquid Crystals and Plastic Crystals, Ref. 14, pp. 48-59.

${ }^{60}$ Lee et al. (Ref. 38) claim that, starting from the same potential as McMillan does, but by doing a better minimization of the free energy, they can get agreement with experimental results for the dependence of the transition temperatures on molecular length. This is false. McMillan's choice for the in- termolecular potential is equivalent, in terms of our theory, to modeling $c_{2}(q)$ as $\left[V_{0} /\left(5 k_{B} T\right)\right] \exp \left(-q^{2} r_{0}^{2} / 4\right)$, where $r_{0}$ is a range parameter and $c_{0}(q)$ as $\delta c_{2}(q)$. Here $V_{0}$ is thought of as independent of the molecular length $d$; hence $T_{N \cdot L}=0.2203 V_{0} / k_{B}$ is independent of $d$. What Lee et al. do (in a way that is not obvious) is to make $V_{0}$ proportional to $\xi_{0}$ $\left(=2 r_{0} / d\right)$. Hence they obtain $T_{N \cdot L} \propto d^{-1}$. As far as we can see this is tantamount to building into the theory the result that is desired.

${ }^{61}$ J. L. Lebowitz and J. W. Perram, Mol. Phys. 50, 1207 (1983).

${ }^{62}$ For such a system, the anisotropic $c^{(2)}\left(\mathbf{r}_{12} ; \widehat{\mathbf{z}}, \hat{\mathbf{z}}\right)$ can be transformed to the direct correlation function of a hardsphere system by a global, anisotropic scale transformation. Hence, as has been pointed out by Harrowell and Oxtoby (Ref. 41), there can be no one-dimensional, i.e., smectic, ordering in a system of fully aligned hard ellipsoids of revolution. 


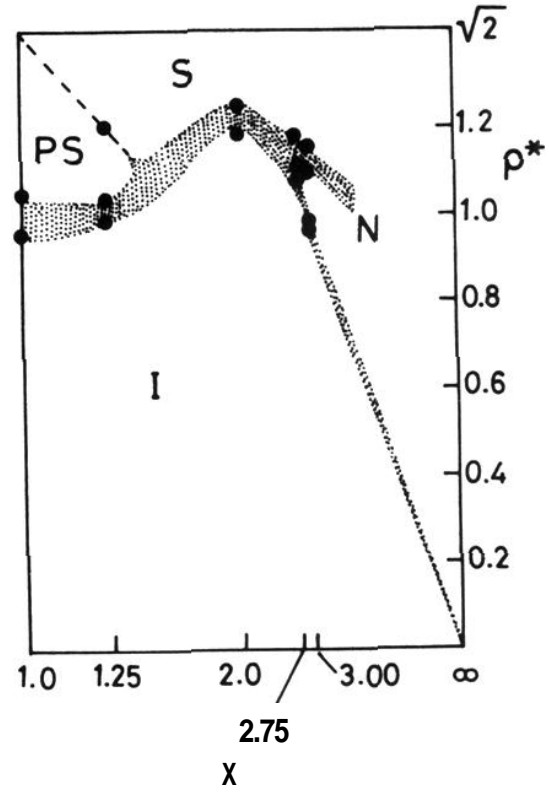

FIG. 3. A part of the phase diagram for a system of hard ellipsoids of revolution as obtained from the molecular-dynamics simulation of Frenkel and Mulder (Ref. 11). The symbols I, $N$, PS, and $S$ represent, respectively, isotropic liquid, nematic, fcc plastic crystal, and (distorted) fcc crystalline phases. $\rho^{*}$ is the reduced density related to the packing fraction 9 through $\eta=(\pi / 6) \rho^{*}$. Shaded areas are regions of two-phase coexistence. 